\title{
Miscibility Behavior of Sphingomyelin with Phytosterol Derivatives by a Langmuir Monolayer Approach
}

\author{
Seiichi Sakamoto, Hiromichi Nakahara and Osamu Shibata* \\ Department of Biophysical Chemistry, Faculty of Pharmaceutical Sciences, Nagasaki International University, 2825-7 Huis Ten Bosch, Sasebo, \\ Nagasaki 859-3298, Japan
}

\begin{abstract}
The miscibility behavior of palmitoyl sphingomyelin (PSM) with phytosterol derivatives of $\beta$-sitosterol (SITO), $\beta$-sitosteryl glucoside (SG), and $\beta$-sitosteryl glucoside palmitate (SGP) was systematically investigated using Langmuir monolayers. The surface pressure $(\pi)$-molecular area $(A)$ and surface potential $(\Delta V)-A$ isotherms for binary PSM/SITO, PSM/SG, and PSM/SGP systems on 0.02 M Tris buffer with $0.13 \mathrm{M} \mathrm{NaCl}\left(\mathrm{pH}\right.$ 7.4) were measured as a function of the molar fraction of PSM $\left(X_{\mathrm{PSM}}\right)$. The surface potentials $(\Delta V)$ of the pure components were analyzed using the three-layer model proposed by Demchak and Fort [J. Colloid Interface Sci. 46 (1974) 191-203]. The contributions of the hydrophilic D-glucose moiety, hydrophobic palmitoyl group, and sphingomyelin group to the vertical component of the dipole moment $\left(\mu_{\perp}\right)$ were evaluated. The thermodynamic quantities, based on the $\pi-A$ isotherms, revealed that PSM interacts attractively with all three phytosterol derivatives in the following order: SITO > SGP > SG. In addition, the two-dimensional phase diagram constructed based on the phase transition pressure $\left(\pi^{\mathrm{eq}}\right)$, from a liquid-expanded to liquid-condensed state, and collapse $\left(\pi^{\mathrm{c}}\right)$ pressure shows that the twocomponent systems are all miscible with each other. The manner of miscibility between PSM/SG and PSM/ SGP systems is found to be opposite in the large $X_{\mathrm{PSM}}$ region. The interaction between the same molecules (PSM-PSM or SG-SG) is stronger than that of the different molecules (PSM-SG) in the PSM/SG system, and vice versa in the PSM/SGP system. These results suggest that the incorporation of a D-glucose and palmitoyl group to a SITO molecule dramatically changes the miscibility behavior with PSM. Observations using fluorescence microscopy imaging also provide insights into miscibility behavior in the monolayer state.
\end{abstract}

Key words: Langmuir monolayer, Miscibility, $\pi-A$ and $\Delta V-A$ isotherm, $N$-palmitoyl-D-erythro-sphingosylphosphorylcholine, Phytosterol derivatives

\section{Introduction}

Lipid rafts have recently been proposed as a microdomain of plasma membranes. They are thought to be a highly dynamic assembly that floats freely within a liquid, disordered bilayer in cell membranes ${ }^{1-3}$. They are composed mainly of sphingolipids (both sphingomyelins (SM) and glycosphingolipids), phospholipids, and sterols. In rafts, it is postulated that the long saturated acyl chains in the sphingolipids tightly interact and are packed with cholesterol (CHOL), resulting in an organization of liquid-ordered $\left(l_{0}\right)$ phases $^{4-7)}$. In contrast, unsaturated phospholipids are loosely packed to form liquid-disordered $\left(l_{\mathrm{d}}\right)$ phases. The different packing properties lead to phase-separated membranes ${ }^{8)}$. Although they are relatively new, lipid rafts have attracted significant attention because they constantly provide dynamic scaffolding for a variety of different cellular processes such as protein trafficking ${ }^{2)}$, signal transduction $^{9,10)}$, CHOL and membrane transport ${ }^{11,12)}$, and calcium homeostasis ${ }^{13)}$.

Plant sterols, or phytosterols, are primarily known as substances that effectively lower the plasma level of lowdensity lipoprotein CHOL, which is an important risk factor for atherosclerosis and cardiovascular diseases ${ }^{14)}$. In addition, phytosterols are naturally occurring in plants and are concentrated in most vegetable oils ${ }^{15}$. In this regard, the demand for phytosterols as a functional food and food ad-

\footnotetext{
*Correspondence to: Osamu Shibata, Department of Biophysical Chemistry, Faculty of Pharmaceutical Sciences, Nagasaki International University; 2825-7 Huis Ten Bosch, Sasebo, Nagasaki 859-3298, Japan

E-mail: wosamu@niu.ac.jp

Accepted May 20, 2013 (received for review April 8, 2013)

Journal of Oleo Science ISSN 1345-8957 print / ISSN 1347-3352 online

http://www.jstage.jst.go.jp/browse/jos/ http://mc.manusriptcentral.com/jjocs
} 
ditive has been increasing worldwide. In human in vivo studies, the intestinal absorption of CHOL is competitively inhibited by phytosterols, resulting in lowering its level in the plasma. This is based on incorporation of phytosterols into the tissues and cellular membranes of erythrocytes because of the structural similarity between them ${ }^{16)}$.

In addition to phytosterols, steryl glycosides $(\mathrm{StG})$ and acyl steryl glycosides (AStG) are also known as major sterol derivatives ${ }^{17-20)}$. StG and AStG both have a broad diversity, which ranges from the type of sterol, sugar moiety, linkage configuration, and number of sugar groups to the acylation of the sugar unit. As the most common form of StG, a sugar monomer, which is pyranose form of D-glucose, is attached to the $3 \beta$-hydroxy group on the $\mathrm{C} 3$ position of $\beta$-sitosterol

(A)

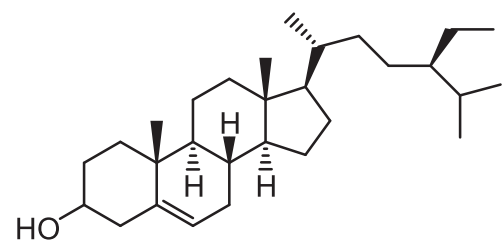

(B)
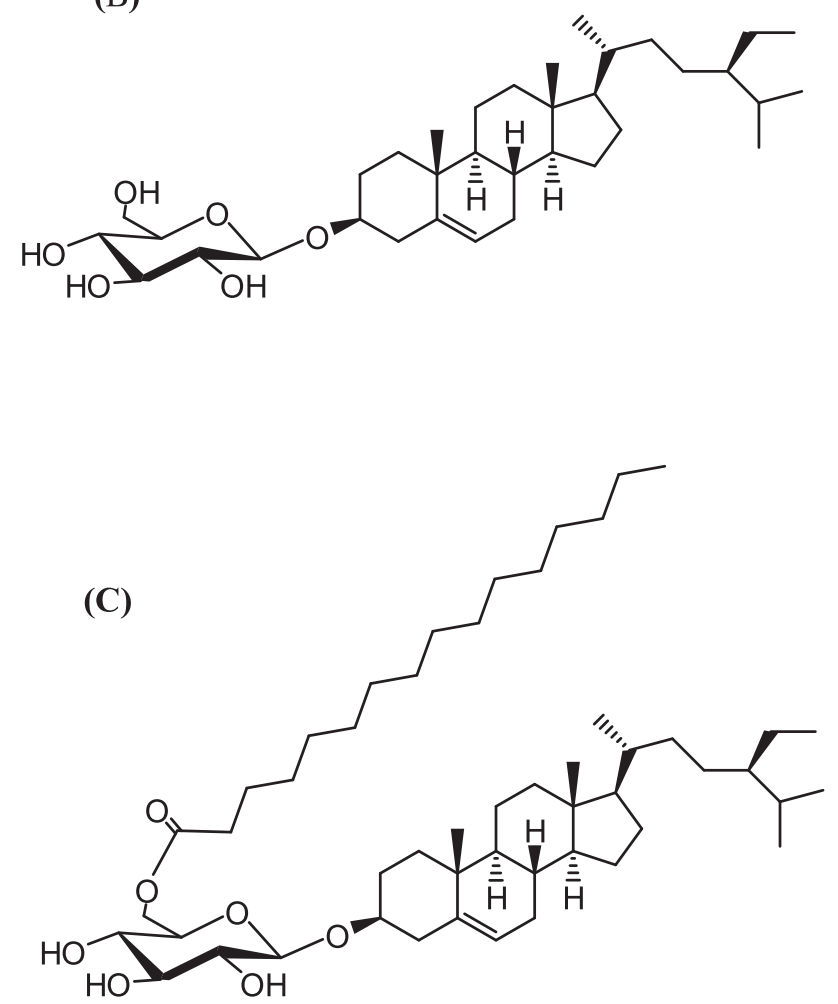

Fig. 1 Chemical structures of (A) $\beta$-sitosterol (SITO), (B) $\beta$-sitosteryl glucoside (SG) and (C) $\beta$-sitosteryl glucoside palmitate (SGP).
(SITO) (Fig. 1(B)), becoming so called $\beta$-sitosteryl glucoside (SG). Generally, acylation of fatty acids mainly occurs at the $\mathrm{C} 6$ position of the sugar moiety. As shown in Fig. 1 (C), $\beta$-sitosteryl glucoside palmitate (SGP) is connected at that position with palmitic acid(16:0), which is the most common fatty acid found in $\mathrm{AStG}^{21)}$. Lin et al. reported that StG and AStG also lower CHOL absorption in humans ${ }^{22,23)}$. However, the effect of such phytosterol derivatives on membrane properties remains unknown, though phytosterols have been reported to modify fluidity, fragility, and deformability of erythrocyte membranes ${ }^{24,25)}$. The interaction between phytosterol derivatives and membranes is of interest from the viewpoint of diet and health food, because they are contained much in a plant only.

The Langmuir monolayer has been widely utilized to elucidate the interaction between a model membrane, mimicking an outer leaflet of plasma membrane, and compounds of interest. Using that method allows easy capture of dynamic formation and destruction of lipid rafts. Up to date, the monolayer interactions between sterol derivatives and phospholipids, such as dipalmitoylphosphatidylcholine $(\mathrm{DPPC})^{26)}$, palmitoylmyristoylphosphatidylcholine $(\mathrm{PMPC})^{26)}$, palmitoyloleoylphosphatidylcholine (POPC $)^{27,28)}$, and sphingolipids of egg-SM ${ }^{29,30)}$ have been extensively reported. In these studies, the condensing effect of sterol derivatives on sphingolipid monolayers was elucidated by adding various kinds of sterol derivatives to egg-SM. As a result, CHOL was found to be much more effective for condensation of aliphatic chains in sphingolipids than in phytoster$\mathrm{ols}^{30)}$. In addition, it was revealed that the SM-sterol interaction is reinforced in a 2:1 proportion. In this manner, miscibility, interaction, and phase behavior in the binary system can be widely discussed by employing Langmuir monolayers. However, there is very little information on SG and SGP, although they are the most common phytosterol derivatives. Furthermore, it is worth noting that little is known about the effect modification of the hydrophilic group will have on the interfacial behavior of an amphiphile, even though the effect of modification on a hydrophobic group has been well investigated ${ }^{26-30)}$. In the present study, the structural differences of SITO, SG, and SGP in the monolayer state were evaluated using a three-layer model, where the dipole moment of the subphase, polar head group, and hydrophobic chain were considered. In addition, the monolayer interactions of palmitoyl SM(PSM) with SITO, SG, and SGP were systematically investigated at the air-water interface from the aspects of thermodynamics, surface potential analyses, and surface morphology.

\subsection{Materials}

$N$-palmitoyl-D-erythro-sphingosylphosphorylcholine (palmitoyl sphingomyelin, PSM; >99\%), (2,4- $\beta$-ethyl) -stigmast-5-en-3 $\beta$-ol ( $\beta$-sitosterol, SITO; >99\%), and 1-palmitoyl-2- \{6- [ (7-nitro-2-1,3-benzoxadiazol-4-yl) 
amino] hexanoyl\}-sn-glycero-3-phosphocholine (NBD-PC; $>99 \%$ ) were purchased from Avanti Polar Lipids, Inc. (Alabaster, AL, USA). $\beta$-Sitosteryl glucoside (SG; >98\%) and $\beta$-sitosteryl glucoside palmitate (SGP; >98\%) were purchased from Matreya, LLC (Pleasant Gap, PA, USA). These lipids were used without further purification or characterization. Chloroform (99.7\%) and methanol (99.8\%)were purchased from Kanto Chemical Co. Inc. (Tokyo, Japan) and nacalai tesque (Kyoto, Japan), respectively, and used as spreading solvents. A chloroform/methanol (2/1, v/v) mixture was used for the preparation of stock solutions for each monolayer $(1.0 \mathrm{mM})$. Tris (hydroxymethyl) aminomethane (Tris), sodium chloride $(\mathrm{NaCl})$, and acetic acid were obtained from nacalai tesque. To remove the surfaceactive organic impurities, $\mathrm{NaCl}$ was heated at $1023 \mathrm{~K}$ for 24 $\mathrm{h}$ before use. Thrice distilled water, with a surface tension of $72.0 \mathrm{mN} \mathrm{m}^{-1}$ at $298.2 \mathrm{~K}$ and electrical resistivity of 18 $\mathrm{M} \Omega \mathrm{cm}$, was used for preparation of a subphase solution.

\subsection{Surface pressure-area isotherms}

A Wilhelmy balance (Mettler Toledo, AG-245), with a resolution of $0.01 \mathrm{mN} \mathrm{m}^{-1}$, was used to measure surface pressure $(\pi)$ against the molecular area $(A)$ for the Langmuir monolayers ${ }^{31-33)}$. The pressure-measuring system was equipped with filter paper (Whatman 541, periphery $=4$ $\mathrm{cm}$ ). The trough was made of Teflon-coated brass (area $=$ $750 \mathrm{~cm}^{2}$ ), and Teflon barriers (both hydrophobic and lipophobic) were used in this study. The $\pi-A$ isotherms were recorded at $298.2 \pm 0.1 \mathrm{~K}$, and the temperature was controlled using a circulating water system. After spreading the samples onto a subphase of a $0.02 \mathrm{M}$ Tris buffer solution, with $0.13 \mathrm{M} \mathrm{NaCl}$ from the organic solvent, the spreading solvents were allowed to evaporate for $15 \mathrm{~min}$ prior to compression. The monolayer was compressed at a speed of $\sim 0.10 \mathrm{~nm}^{2}$ per molecule per minute. The standard deviations (SD) for the molecular area and surface pressure were $\sim 0.01 \mathrm{~nm}^{2}$ and $\sim 0.1 \mathrm{mN} \mathrm{m}^{-1}$, respectively. The $\mathrm{pH}$ of the subphase was adjusted to 7.4 with an adequate amount of acetic acid.

\subsection{Surface potential-area isotherms}

The surface potential $(\Delta V)$ was measured simultaneously with the surface pressure when the monolayer was compressed at the air-water interface. It was monitored using an ionizing ${ }^{241} \mathrm{Am}$ electrode $1-2 \mathrm{~mm}$ above the interface, while a reference electrode was dipped into the subphase. An electrometer (Keithley 614) was used to measure the surface potential. The SD for the surface potential was $\sim 5$ $\mathrm{mV}^{31-33)}$.

\subsection{Brewster angle microscopy (BAM)}

The monolayer was directly visualized using a Brewster angle microscope (KSV Optrel BAM 300, KSV Instruments, Finland) coupled to a commercially available film balance
system(KSV Minitrough, KSV Instruments, Finland)at $298.2 \pm 0.1 \mathrm{~K}$. In the BAM experiments, a $20 \mathrm{~mW} \mathrm{He}-\mathrm{Ne}$ laser, at a wavelength of $632.8 \mathrm{~nm}$, was used as the light source. A Glan-Thompson polarizer was placed between the laser and minitrough to provide $p$-polarized light at the Brewster angle $\left(53.1^{\circ}\right)$. A $10 \times$ objective lens allowed a lateral resolution of $\sim 2 \mu \mathrm{m}$. The angle of the incident beam to the air-water interface was fixed to the Brewster angle, and the reflected beam was recorded with a highgrade charge-coupled device (CCD) camera (EHDkamPro02, EHD Imaging GmbH, Germany). The BAM image was digitally saved on a computer hard disk ${ }^{33)}$.

\subsection{Fluorescence microscopy (FM)}

The film balance system (KSV Minitrough, KSV Instruments, Finland) was mounted onto the stage of an Olympus microscope BX51W1 (Tokyo, Japan) equipped with a $100 \mathrm{~W}$ mercury lamp (USH-1030L), an objective lens (SLMPlan 50 $\times$, SLMPlanFI $20 \times$, working distance $=15 \mathrm{~mm}$ ), and a 3 CCD camera control unit(IKTU51CU, Toshiba, Japan). The $z$-directional focus on the monolayer was adjusted using an automation controller (MAC 5000, Ludl Electronic Products Ltd., NY). The stock solution for FM was prepared by addition of $1 \mathrm{~mol} \%$ of the fluorescent probe, NBD-PC. Images taken under an excitation wavelength of $460 \mathrm{~nm}$ and an emission wavelength of $534 \mathrm{~nm}$ were directly recorded to the hard disk via an online image processor (DVgate Plus; Sony, Tokyo, Japan) connected to the microscope. Image processing and analysis were done using Adobe Photoshop Elements ver. 9.0 (Adobe System Incorporated, CA). The total quantity of ordered domains (dark contrast regions) was evaluated and expressed as a percentage per frame by dividing the respective frames into dark and bright regions $^{31,32)}$.

\subsection{Isotherm behavior for binary monolayers of PSM and phytosterol derivatives (SITO, SG, and SGP)}

The surface pressure $(\pi)$-molecular area $(A)$ and surface potential $(\Delta V)-A$ isotherms for pure PSM, SITO, SG, and SGP were measured on $0.02 \mathrm{M}$ Tris buffer with $0.13 \mathrm{M} \mathrm{NaCl}$ ( $\mathrm{pH}$ 7.4) at $298.2 \mathrm{~K}$ (Fig. 2). The pure PSM monolayer (curve 3) possesses a phase transition pressure $\left(\pi^{\mathrm{eq}}\right)$ of $\sim$ $21 \mathrm{mN} \mathrm{m}^{-1}$ at $298.2 \mathrm{~K}$, at which point the monolayer state changes from a liquid-expanded (LE) to a liquid-condensed (LC) phase (indicated by an arrow). Monolayer collapse, when monolayers begin to transform into a bulk phase, is observed at $\sim 57 \mathrm{mN} \mathrm{m}^{-1}$ with further compression. The $\pi-A$ isotherms of SITO (curve 1), SG (curve 2), and SGP (curve 4) show that they form a typical LC monolayer with monolayer collapse pressures $\left(\pi^{c}\right)$ of $\sim 40.6, \sim 51.2$, and $\sim$ $45.3 \mathrm{mN} \mathrm{m}^{-1}$, respectively. The $\pi^{\mathrm{c}}$ value of SG increases, but it is generally estimated that the incorporation of Dglucose leads to a LE monolayer state. These results indicate that the incorporation of D-glucose into the head 


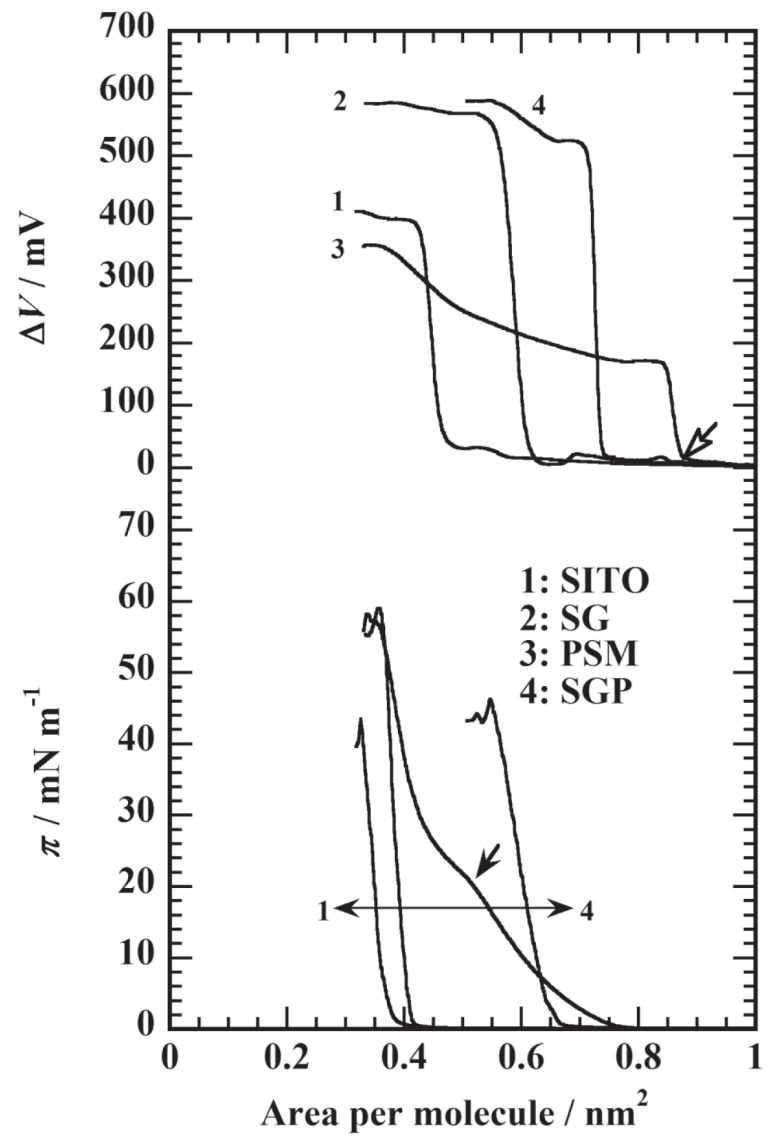

Fig. 2 The $\pi-A$ and $\Delta V-A$ isotherms of pure PSM, SITO, SG, and SGP monolayers spread on 0.02 $\mathrm{M}$ Tris buffer containing $0.13 \mathrm{M} \mathrm{NaCl}$ at 298.2 $\mathrm{K}$ (pH 7.4). The open-headed arrow in the $\Delta V-A$ isotherms represents the transition point from gas state of monolayers, and the solid arrow in the $\pi$ - $A$ isotherms indicate an LE/LC phase transition at $\sim 21 \mathrm{mN} \mathrm{m}^{-1}$ of pure PSM.

group of SITO unexpectedly enhances the stability of the monolayers upon compression. In addition, further introduction of a palmitoyl group into SG induces a decrease in $\pi^{c}$. It is assumed that the hydrophobic steroid backbone plays an essential role in the dominating $\pi^{\mathrm{c}}$ value, because the $\pi^{c}$ value is not affected much by length modification or the carbon-carbon binding manner of the sterol backbone side chain ${ }^{26,27,29,30)}$. On the other hand, modification of the hydroxyl group $(-\mathrm{OH})$ at the C3 position causes changes in the $\pi^{\mathrm{c}}$ value, as mentioned above. This may be accounted for by the hydration degree of the hydrophilic group in the subphase. SG contains D-glucose as a hydrophilic group, and thus it attracts water molecules more strongly than SITO, resulting in an increase in the $\pi^{c}$ value for SG. In contrast, the hydration degree of SGP is smaller than that of SG because of the incorporation of a palmitoyl group into the D-glucose of SG, which causes the decreased $\pi^{c}$ value for SGP. As for a limiting area of SITO and SG, they have respectively $\sim 0.38 \mathrm{~nm}^{2}$ which is found to be identical to that of $\mathrm{CHOL}\left(\sim 0.38 \mathrm{~nm}^{2}\right)^{34)}$, and $\sim 0.41 \mathrm{~nm}^{2}$ which is in agreement with that reported previously ${ }^{35)}$. In the SGP monolayer, the limiting area is $\sim 0.64 \mathrm{~nm}^{2}$. The difference in limiting area between $\mathrm{SG}\left(\sim 0.41 \mathrm{~nm}^{2}\right)$ and $\mathrm{SGP}(\sim 0.64$ $\left.\mathrm{nm}^{2}\right)$ is $\sim 0.23 \mathrm{~nm}^{2}$. This value is almost the same as the cross-sectional area, $\sim 0.19 \mathrm{~nm}^{2}$, of one saturated aliphatic chain. This means that the palmitoyl chain of SGP is thought to be positioned beside the SG moiety in the closepacked state, keeping a small cavity $\left(0.04 \mathrm{~nm}^{2}\right)$ where the palmitoyl chain can move.

The $\Delta V$ value is a measure of the electrostatic field gradient vertical to the surface, and varies considerably with the dipole density of the molecular surface. Thus, the $\Delta V$ value is highly sensitive to changes in the monolayer orientation and conformation. The $\Delta V-A$ isotherm reflects the phase transition from a gas to a liquid state, where the orientation of the hydrophobic chain starts to change (indicated by an open-headed arrow in Fig. 2). The $\Delta V$ values of all the monolayers positively vary upon compression, which means that the monolayer orientation is improved in the direction of the normal surface normal ${ }^{36,37)}$. The maximum $\Delta V$ values for SITO, SG, and SGP monolayers are $\sim 410$ $\mathrm{mV}, \sim 590 \mathrm{mV}$, and $\sim 590 \mathrm{mV}$, respectively. $\Delta V$ values depend on the hydrophilic moiety, terminal hydrophobic moiety, and subphase ${ }^{38)}$. The contribution of the D-glucose moiety to the $\Delta V$ values is estimated to be $\sim 180 \mathrm{mV}$, assuming that the hydrophobic parts of SITO and SG are similarly packed. Interestingly, the maximum $\Delta V$ value of $\mathrm{SG}(\sim 590 \mathrm{mV})$ is the same as that of $\mathrm{SGP}(\sim 590 \mathrm{mV})$. These results are derived from the terminal moiety of the hydrophobic part, which generally affects the $\Delta V$ value in the close-packed state. The terminal hydrophobic moiety of SG is the methyl $\left(-\mathrm{CH}_{3}\right)$ group. Similarly, as for SGP, the methyl group, derived from the palmitoyl group, is located at the terminal of monolayers. Thus, the contribution of the palmitoyl-group introduction in SGP to the $\Delta V$ value is considered to be negligible.

The $\pi-A$ and $\Delta V-A$ isotherms for binary (PSM/SITO, $\mathrm{PSM} / \mathrm{SG}$, and PSM/SGP) systems have been measured at 298.2 K to reveal the interactions of PSM with phytosterol derivatives (Fig. 3 ). In the binary PSM/SITO system(Fig. 3 (A) ), the $\pi-A$ isotherms overlap at $0 \leq X_{\mathrm{PSM}} \leq 0.5$. This behavior can be discussed in terms of the additivity rule for molecular areas ${ }^{39,40)}$ (Fig. 4). The dashed line is drawn according to the additivity rule, and the solid points represent the experimental value. Negative deviation from linearity implies that PSM attracts SITO to a large cavity, which is produced by like a corn-motion of PSM, and then wraps the SITO molecule. In addition, the experimental molecular area shows the almost constant value over the range of $0 \leq X_{\mathrm{PSM}} \leq 0.5$, which results from the overlap observed in the $\pi-A$ isotherms (curves 1-4 in Fig. 3(A)). 

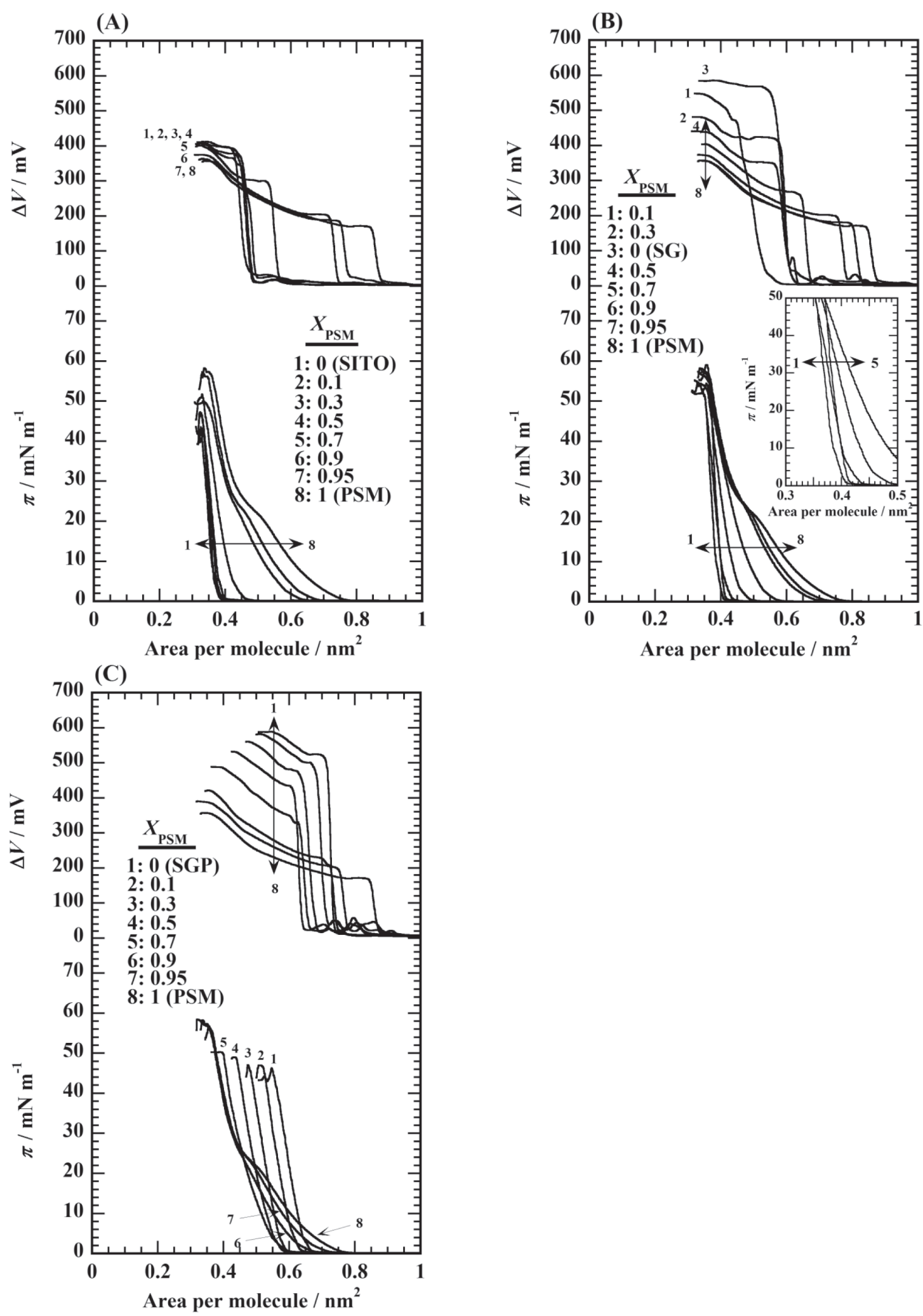

Fig. 3 The $\pi$ - $A$ and $\Delta V-A$ isotherms of binary monolayers at the air/water interface on $0.02 \mathrm{M}$ Tris buffer containing 0.13 $\mathrm{M} \mathrm{NaCl}$ (pH 7.4) at $298.2 \mathrm{~K}$ formed by PSM and phytosterol derivatives: (A) PSM/SITO, (B) PSM/SG, and (C) PSM/SGP monolayers. An inset in the Fig. 3 (B) represents enlarged $\pi$ - $A$ isotherms in the surface pressure region of $0 \leq \pi \leq 50 \mathrm{mN} \mathrm{m}^{-1}\left(0.3 \leq \mathrm{A} \leq 0.5 \mathrm{~nm}^{2}\right)$. 
(A)

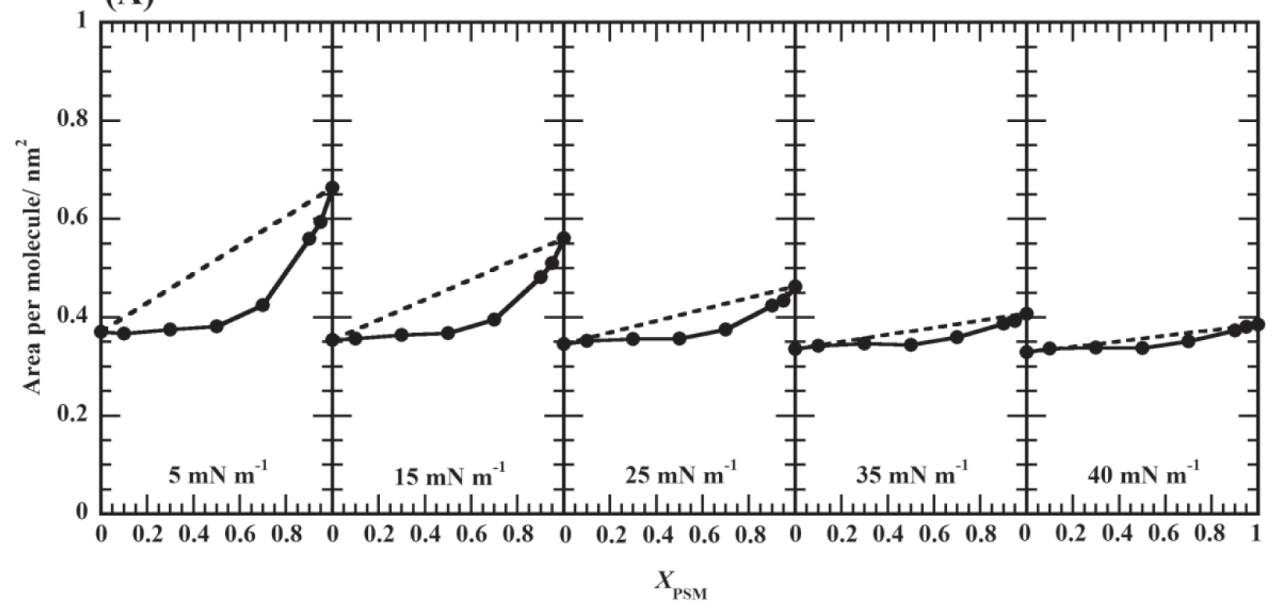

(B)

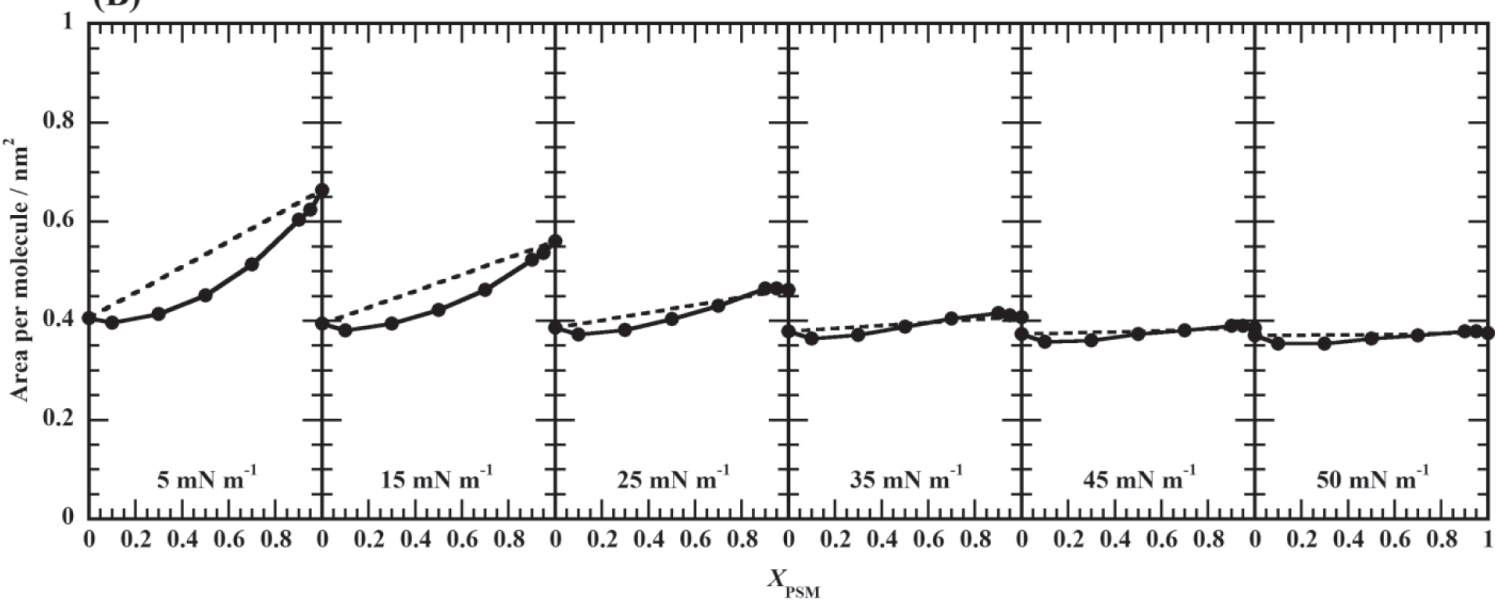

(C)

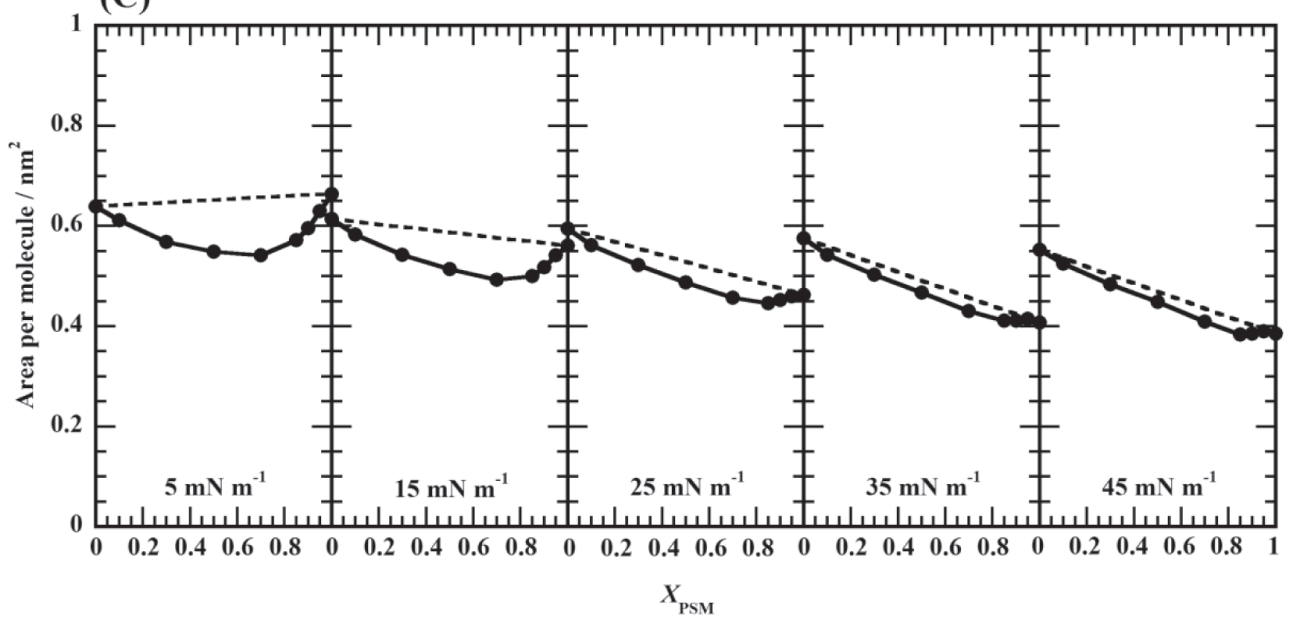

Fig. 4 Mean molecular areas (A) of binary (A) PSM/SITO, (B) PSM/SG, and (C) PSM/SGP systems as a function of $X_{\mathrm{PSM}}$ at different surface pressures. The dashed lines were evaluated by the additivity rule, and the solid circles represent experimental values. 
This overlap indicates that SITO has a condensing effect up to $X_{\mathrm{PSM}}=0.5$. As for the collapse, the $\pi^{\mathrm{c}}$ values in the binary PSM/SITO system gradually increases with increasing $X_{\mathrm{PSM}}(\mathrm{Fig} .7(\mathrm{~A}))$. In the $\Delta V-A$ isotherms, the maximum $\Delta V$ value at $0 \leq X_{\mathrm{PSM}} \leq 0.5$ converges on $\sim 408 \mathrm{mV}$ (curves $1-4)$. The values decrease with an increase in $X_{\mathrm{PSM}}$, where maximum $\Delta V$ value of PSM is $\sim 354 \mathrm{mV}$ (Fig. 2). These results indicate that the PSM/SITO monolayer, over the range of $0.1 \leq X_{\mathrm{PSM}} \leq 0.5$, is similar to the single SITO monolayer. In the case of the binary PSM/CHOL system, the same behavior was observed; the additional effect of PSM disappears in the specific $X_{\mathrm{PSM}}$ range $^{34)}$. Considering the structural similarity between SITO and CHOL, the bulkiness in the hydrophobic moieties (or steroid backbone) is deeply related to such phenomena.

For the binary PSM/SG system(Fig. 3(B)), the pattern of the $\pi-A$ isotherms is similar to the patterns in the PSM/ SITO system. However, the behavior at $X_{\mathrm{PSM}}=0.1$ is found to be unique. The $\pi-A$ isotherm at $X_{\mathrm{PSM}}=0.1$ (curve 1) shifts to smaller areas compared to pure SG monolayers (Fig. 2), even though the other isotherms vary between pure PSM and SG. This suggests that the small amounts of $\operatorname{PSM}\left(X_{\mathrm{PSM}}=0.1\right)$ provoke a stronger condensation than that seen at $0.3 \leq X_{\mathrm{PSM}} \leq 0.95$. Because the extrapolated molecular area of $\operatorname{PSM}\left(0.48 \mathrm{~nm}^{2}\right)$, the molecular area of the ordered-state part on the $\pi-A$ isotherm at $\pi=0$, is larger than the limiting area of $\mathrm{SG}\left(0.41 \mathrm{~nm}^{2}\right)$, a condensing effect (attractive interaction) seems to be generated to have smaller limiting area at a specific ratio of $X_{\mathrm{PSM}}=0.1$. The $\pi^{\mathrm{c}}$ values in the binary PSM/SG system gradually increase by $\sim 5.3$ $\mathrm{mN} \mathrm{m}^{-1}$ as $X_{\mathrm{PSM}}$ increases (Fig. $7(\mathrm{~B})$ ). In the $\Delta V-A$ isotherms, the maximum $\Delta V$ value decreases from $\sim 590 \mathrm{mV}$ (SG) to $\sim 354 \mathrm{mV}$ (PSM) as $X_{\mathrm{PSM}}$ increases. When a jump in the $\Delta V$ value, corresponding to the transition from the gas phase to the liquid condensed phase, is focused on, the jump at $X_{\mathrm{PSM}}=0.1$ (curve 1) appears at smaller areas compared with other isotherms (curves 2-8). This corresponds to the $\pi-A$ isotherm (curve 1) mentioned above. In Fig. 4 (B), the degree of negative deviation from linearity is smaller than in the PSM/SITO system, especially up to 35 $\mathrm{mN} \mathrm{m}^{-1}$. Although PSM interacts attractively with SG, as well as SITO, the insertion of the SG steroid backbone into the cavity of PSM in the PSM/SG system is likely prevented by the D-glucose moiety, more so than in the PSM/SITO system.

In the case of the binary PSM/SGP system(Fig. 3(C)), the $\pi-A$ isotherms shift to a smaller area with increasing $X_{\mathrm{PSM}}$. In addition, the $\pi^{\mathrm{c}}$ values gradually increase with increasing $X_{\text {PSM }}($ Fig. $7(\mathrm{C}))$. Interestingly, several $\pi-A$ isotherms (curves 6-7) overlap with those for pure PSM (curve 8) above $\sim 29.0 \mathrm{mN} \mathrm{m}^{-1}$, but they are different below it. Among the $\pi-A$ isotherms (curves 6-8) below $29.0 \mathrm{mN} \mathrm{m}^{-1}$, the pure PSM isotherm (curve 8) has the largest mean area. The $\Delta V-A$ isotherms display a similar pattern to those for the PSM/SG system, which exhibits a decrease in the maximum $\Delta V$ value from $\sim 590 \mathrm{mV}$ (SGP) to $\sim 354 \mathrm{mV}$ (PSM) as $X_{\mathrm{PSM}}$ increases. Concerning the $A-$ $X_{\text {PSM }}$ plots (Fig. 4(C)), the degree of negative deviation from ideality is larger than that seen in the PSM/SG system (Fig. 4(B)). This suggests that PSM interacts more strongly with SGP than SG, because the saturated palmitoyl group in SGP is considered to enhance cohesive attraction of double-saturated acyl chains in PSM.

\subsection{Contribution of $\omega$-groups and polar head groups to the dipole moment for phytosterol derivatives}

To investigate the structural differences in a pure monolayer, the surface potential of monolayers on $0.02 \mathrm{M}$ Tris buffer with $0.13 \mathrm{M} \mathrm{NaCl}(\mathrm{pH} 7.4$ ) was analyzed using the three-layer model proposed by Demchak and Fort ${ }^{41}$, which is based on the earlier model of Davies and Rideal ${ }^{42}$. This model postulates independent contributions from the subphase (layer 1), polar head group (layer 2), and hydrophobic chain (layer 3). Independent dipole moments and effective local dielectric constants are attributed to each of the three layers. Other models such as the Helmholtz model and the Vogel-Möbius model are also available ${ }^{43)}$. These different models were recently reviewed ${ }^{44)}$. The conclusion of the review was that, despite the limitations of each, the Demchak and Fort model provides good agreement between $\mu_{\perp}$ values estimated from the monolayer's surface potentials and those determined from measurements on the bulk material, for various aliphatic compounds.

The estimation of $\mu_{\perp}$ (the vertical components of the dipole moment on the plane of the monolayer) for polar head groups and hydrocarbon chains, using the Demchak and Fort model, assumes a close-packed Langmuir monolayer ${ }^{41,44}$. Application of this model to the PSM LC/LE monolayer may lead to a rough estimation. However, if the closest-packed PSM monolayer is applied to this model, it may lead to a useful estimate, which can help provide a qualitative explanation of surface potential behavior.

Thus, the experimental values of $\mu_{\perp}$ in the most condensed state of the monolayer have been compared with those calculated using the three-layer model equation:

$$
\mu_{\perp \text { Calc }}=\left(\frac{\mu_{1}}{\varepsilon_{1}}\right)+\left(\frac{\mu_{2}}{\varepsilon_{2}}\right)+\left(\frac{\mu_{3}}{\varepsilon_{3}}\right)
$$

where $\mu_{1} / \varepsilon_{1}, \mu_{2} / \varepsilon_{2}$, and $\mu_{3} / \varepsilon_{3}$ are the contributions of the subphase, polar head group, and hydrophobic chain group, respectively.

The initial set of values proposed by Demchak and Fort $\left(\mu_{1} / \varepsilon_{1}=0.040 \mathrm{D}, \varepsilon_{2}=7.6\right.$, and $\left.\varepsilon_{3}=5.3\right)$ were determined for monolayers made from terphenyl derivatives and octadecylnitrile. Another set of values were determined by Petrov, Polymerropoulos, and Möhwald $\left(\mu_{1} / \varepsilon_{1}=0.025 \mathrm{D}, \varepsilon_{2}=7.6\right.$, and $\varepsilon_{3}=4.2$ ) for monolayers of $n$-heptanol and 16-bromohexadecanol $^{45)}$ and by Taylor and Oliveira $\left(\mu_{1} / \varepsilon_{1}=-0.065\right.$ 


\section{S. Sakamoto, H. Nakahara and O. Shibata}

$\mathrm{D}, \varepsilon_{2}=6.4$, and $\varepsilon_{3}=2.8$ ) for monolayers of $\omega$-halogenated fatty acids and amines ${ }^{44)}$. Carboxylic and hydroxyl groups have already been determined using the Demchak and Fort model $^{41)}$.

To determine a set of parameters that fit the experimental conditions using $0.02 \mathrm{M}$ Tris buffer with $0.13 \mathrm{M} \mathrm{NaCl}$ ( $\mathrm{pH}$ 7.4), the selection of parameter values was done using those for the standard sample, in which general stearic $\operatorname{acid}(\mathrm{SA})$ is used. The subphase condition in this study is $\mathrm{pH}$ 7.4. The main difference between fatty acids and fatty alcohols is the head group structure; the acids are sensitive to influences from the subphase $\mathrm{pH}$. A carboxyl group in saturated long-chain acids has a value of 5.1-5.7 for its $\mathrm{pKa}^{46)}$. Thus, at $\mathrm{pH} 7.4$, the carboxyl group is partially dissociated (30-44\%) and negatively charged ${ }^{32}$. On the other hand, alcohols are extremely weak acids and only dissociate under strong basic conditions, and there was no evidence of hydroxyl group dissociation.

In this case, hexadecanol (HD) was used for the standard sample shown in Eq. (2). The data are listed in Table 1. The experimental values of the surface dipole moment for HD used to determine the set of the parameters were as follows:

$$
\mu_{\perp}{ }^{\mathrm{HD}}=\left(\frac{\mu_{1}}{\varepsilon_{1}}\right)+\left(\frac{\mu_{2}{ }^{\mathrm{OH}}}{\varepsilon_{2}}\right)+\left(\frac{\mu_{3}{ }^{\mathrm{CH}_{3}}}{\varepsilon_{3}}\right)=0.19 \mathrm{D}
$$

where it was assumed that the $\mathrm{C}-\mathrm{X}$ dipole of the terminal $-\mathrm{CH}_{2} \mathrm{X}$ moiety (where $\mathrm{X}$ is hydrogen) was inclined at half the tetrahedral angle (i.e., $54^{\circ} 44^{\prime}$ ) with respect to the water surface, as suggested by Bernett et $a l^{47)}$ and that the group moments had the values given by Smyth $^{48)}$. In addition, it was assumed that the C-H group moment was 0.40 $\mathrm{D}$, the carbon being negatively charged ${ }^{49)}$. The contribution of the terminal methyl group was $0.33 \mathrm{D}^{41}$. Next, the contribution of $\mu_{2}$ to the surface dipole moment was proposed for the different conformations of the $\mathrm{OH}$ group: $\mu_{2}(\mathrm{OH}-$ gauche $)=1.00 \mathrm{D}, \mu_{2}(\mathrm{OH}-$ trans $)=-0.63 \mathrm{D}$, and $\mu_{2}(\mathrm{OH}-$ free $)=0.18 \mathrm{D}$. In this study the $\mu_{2}(\mathrm{OH}$-gauche $)=1.00 \mathrm{D}$ was employed, as many studies support the gauche conformation for condensed alkanol monolayers ${ }^{41)}$. This gives the result $\mu_{1} / \varepsilon_{1}=-0.083 \mathrm{D}$, which is a little bit different from Taylor and Oliveira's parameter, $\mu_{1} / \varepsilon_{1}=-0.065 \mathrm{D}$. The difference may come from variations in experimental conditions such as substrate composition(buffer solution, electrolyte, and pH), compression rate, and so forth.

As a result, these parameter values had good agreement between calculated and experimental values for dipole moments measured on a saline phase. A combination of set values was used $\left(\mu_{1} / \varepsilon_{1}=-0.083 \mathrm{D}, \varepsilon_{2}=6.4, \mu_{2}\right.$ (OH-gauche) $=1.00 \mathrm{D}, \mu_{3}=0.33 \mathrm{D}$, and $\varepsilon_{3}=2.8$ for $\left.\mathrm{CH}_{3}\right)$. Then the contribution of the hydrophilic group in the phytosterol derivatives was determined by comparing them with the steroid structure of cholesterol.

$$
\begin{aligned}
& \mu_{\perp}{ }^{\mathrm{CHOL}}=\left(\frac{\mu_{1}}{\varepsilon_{1}}\right)+\left(\frac{\mu_{2}{ }^{\mathrm{OH}}}{\varepsilon_{2}}\right)+\left(\frac{\mu_{3}{ }^{\mathrm{CHOL}}}{\varepsilon_{3}}\right)=0.36 \mathrm{D} \\
& \mu_{\perp}{ }^{\mathrm{SITO}}=\left(\frac{\mu_{1}}{\varepsilon_{1}}\right)+\left(\frac{\mu_{2}{ }^{\mathrm{OH}}}{\varepsilon_{2}}\right)+\left(\frac{\mu_{3}^{\mathrm{SITO}}}{\varepsilon_{3}}\right)=0.36 \mathrm{D}
\end{aligned}
$$

From Eqs. (3) and (4), the $\mu_{3}{ }^{\mathrm{CHOL}}=0.81 \mathrm{D}$ and $\mu_{3}{ }^{\mathrm{SITO}}=0.80$ $\mathrm{D}$ values can be obtained. From this result, the contribution of the ethyl group at C24 in SITO to the dipole moment is estimated to be negligible.

Next, from Eq. (5), the contribution from the hydrophilic group of the glycoside can be derived, that is, $\mu_{2}^{\mathrm{D}-\mathrm{Gl}}=2.5 \mathrm{D}$.

$$
\mu_{\perp}^{\mathrm{SG}}=\left(\frac{\mu_{1}}{\varepsilon_{1}}\right)+\left(\frac{\mu_{2}^{\mathrm{D}-\mathrm{Gl}}}{\varepsilon_{2}}\right)+\left(\frac{\mu_{3}^{\mathrm{SITO}}}{\varepsilon_{3}}\right)=0.59 \mathrm{D}
$$

For SGP molecules, the contribution of the hydrophobic steroid segment was evaluated using Eq. (6) (see Table 1). The contribution of $\mu_{3}$ to the surface dipole moment has been proposed for the different chain in the sitosteryl group $\left[\mu_{3}{ }^{\text {SITO }}=0.80 \mathrm{D}\right]$ and the palmitoyl part $\left[\mu_{3}{ }^{\mathrm{C} 16}=0.33\right.$ D]. Then the contribution of $\mu_{2}{ }^{\text {SGP }}$ to the surface dipole

Table 1 Surface potential data used for dipole moment evaluation.

\begin{tabular}{cccc}
\hline Sample & $A\left(\mathrm{~nm}^{2}\right)$ & $\Delta V(\mathrm{mV})$ & $\mu_{\perp}(\mathrm{D})$ \\
\hline Hexadecanol (HD) & 0.179 & $402 \pm 5$ & 0.191 \\
Choresterol (CHOL) & 0.341 & $401 \pm 5$ & 0.364 \\
SITO & 0.329 & $410 \pm 5$ & 0.358 \\
SG & 0.376 & $586 \pm 5$ & 0.585 \\
SGP & 0.564 & $583 \pm 5$ & 0.873 \\
PSM & 0.396 & $349 \pm 5$ & 0.352 \\
DPPC & 0.399 & $551 \pm 5$ & 0.584 \\
\hline
\end{tabular}

$A$ is the molecular surface area at the close-packed high pressure portion of the $\pi$ - $A$ isotherms and $\Delta V$ is obtained at the point. $\mu_{\perp}$ is total dipole moment and the subphase was 0.02 $\mathrm{M}$ Tris buffer with $0.13 \mathrm{M} \mathrm{NaCl}(\mathrm{pH} 7.4)$ at $298.2 \mathrm{~K}$ in all cases. 
moment was evaluated to be $\mu_{2}^{\text {SGP\#1 }}=5.9$ D. However, judging from the $\pi-A$ isotherm, the extrapolated area of SGP is larger, even though the addition of the palmitoyl part area is taken into account. Next, the contribution of $\mu_{3}$ to the surface dipole moment was proposed for the different chain of the sitosteryl group $\left[\mu_{3}^{\mathrm{SITO}}=0.80 \mathrm{D}\right]$, and the palmitoyl part behaves as a sitosteryl group $\left[\mu_{3}{ }^{\mathrm{Cl}}=0.80 \mathrm{D}\right]$. As a result, the contribution of $\mu_{2}^{\mathrm{SGP}}$ to the surface dipole moment was evaluated to be $\mu_{2}^{\text {SGP\#2 }}=2.5 \mathrm{D}$. On the other hand, in general, saturated hydrophobic long-chain is introduced into a molecule, monolayer stability is typically increased. In this case, the collapse pressure $\left(\pi^{c}\right)$ is bigger than the former structure. However, $\pi^{c}$ in SGP is almost the same as that in SITO. Considering the above, the contribution of $\mu_{2}{ }^{\mathrm{SGP}}$ to the surface dipole moment in the SGP molecule is evaluated to be an average of $\mu_{2}^{\text {SGP\#1 }}$ and $\mu_{2}^{\text {SGP\#2 }}$. Its value is $\mu_{2}^{\mathrm{SGP}}=4.2 \mathrm{D}$.

$$
\mu_{\perp}^{\mathrm{SGP}}=\left(\frac{\mu_{1}}{\varepsilon_{1}}\right)+\left(\frac{\mu_{2}^{\mathrm{SGP}}}{\varepsilon_{2}}\right)+\left(\frac{\mu_{3}^{\mathrm{STTO} / \mathrm{C} 16}}{\varepsilon_{3}}\right)=0.87 \mathrm{D}
$$

Next, for the PSM molecule, the contribution of the hydrophilic part was evaluated using Eq. (7) (see Table 1).

$$
\mu_{\perp}^{\mathrm{PSM}}=\left(\frac{\mu_{1}}{\varepsilon_{1}}\right)+\left(\frac{\mu_{2}^{\mathrm{SPC}}}{\varepsilon_{2}}\right)+\left(\frac{\mu_{3}{ }^{\mathrm{CH}_{3}}}{\varepsilon_{3}}\right)=0.35 \mathrm{D}
$$

The contribution of $\mu_{2}{ }^{\text {SPC }}$ to the surface dipole moment in the PSM molecule was evaluated as $\mu_{2}^{\mathrm{SPC}}=1.3 \mathrm{D}$.

Finally, to ensure the suitability of this set of parameters, the contribution of the polar head group to the dipole moment for DPPC was rechecked:

$$
\mu_{\perp}{ }^{\mathrm{DPPC}}=\left(\frac{\mu_{1}}{\varepsilon_{1}}\right)+\left(\frac{\mu_{2}^{\mathrm{PC}}}{\varepsilon_{2}}\right)+\left(\frac{\mu_{3}{ }^{\mathrm{CH}_{3}}}{\varepsilon_{3}}\right)=0.58 \mathrm{D}
$$

The contribution of $\mu_{2}{ }^{\mathrm{PC}}$ to the surface dipole moment in the DPPC molecule was evaluated as $\mu_{2}^{\mathrm{PC}}=2.8 \mathrm{D}$ for DPPC. This value for DPPC is in good agreement with the value reported by Taylor et al., ${ }^{44)}$ (2.44 D for DPPC).

Using the experimentally determined $\Delta V$ values (see Table 1$)$, and assuming a set of values $\left(\mu_{1} / \varepsilon_{1}=-0.083 \mathrm{D}, \varepsilon_{2}\right.$ $=6.4,(\mathrm{OH}$-gauche $)=1.00 \mathrm{D}, \mu_{3}=0.33 \mathrm{D}$ for $\mathrm{CH}_{3}$, and $\varepsilon_{3}=$ 2.8 for $\mathrm{CH}_{3}$ ), the following values were obtained using the above equations: $\mu_{3}{ }^{\text {SITO }}=0.80 \mathrm{D}$ for SITO, $\mu_{2}{ }^{\text {D-Gl }}=2.5 \mathrm{D}$ for $\mathrm{SG}, \mu_{2}{ }^{\mathrm{SGP}}=4.2 \mathrm{D}$ and $\mu_{3}{ }^{\mathrm{SGP}}=0.49 \mathrm{D}$ for SGP, and $\mu_{2}^{\mathrm{SPC}}=1.3$ $\mathrm{D}$ for PSM. These results clearly reflect the contributions to the dipole moment from the hydrophilic and hydrophobic part in each molecule.

\subsection{Excess Gibbs free energy}

The excess Gibbs free energy of mixing, $\Delta G^{\text {exc }}$, reveals information about lipid-lipid interactions in a binary monolayer system. The $\Delta G^{\text {exc }}$ values for the three systems were calculated using the following equation: ${ }^{50,51)}$,

$$
\Delta G^{\mathrm{exc}}=\int_{0}^{\pi}\left(A_{12}-X_{1} A_{1}-X_{2} A_{2}\right) \mathrm{d} \pi
$$

where $A_{i}$ and $X_{i}$ are the area per molecule and molar fraction of component $i$, respectively, and $A_{12}$ is the mean area per molecule in a mixture of components 1 and 2 . In the case of ideal mixing between the two components, $\Delta G^{\text {exc }}=$ $0^{39,40)}$. A negative $\Delta G^{\mathrm{exc}}$ value indicates an attractive interaction between two components. The variations in $\Delta G^{\mathrm{exc}}$ against $X_{\mathrm{PSM}}$ for PSM/SITO, PSM/SG, and PSM/SGP monolayers, at typical surface pressures, are shown in Fig. 5. In the PSM/SITO system (Fig. 5(A)), the $\Delta G^{\text {exc }}$ values are negative over the whole range of surface pressures, which indicates that the interaction between PSM and SITO is more attractive than the PSM-PSM and SITO-SITO interactions. Obviously, these values decrease with increasing surface pressure. The monolayer at $X_{\mathrm{PSM}}=0.7$ is the minimum $\Delta G^{\text {exc }}$ value over the entire surface pressure range, and corresponds to the binary PSM/CHOL system, as reported in a previous paper ${ }^{34)}$. Furthermore, this result agrees with reports on the interaction between SM and CHOL derivatives (CHOL, SITO, and stigmasterol) ${ }^{29}$ ). Figure $5(\mathrm{~B})$ shows $\Delta G^{\text {exc }}-X_{\mathrm{PSM}}$ curves for the PSM/SG system. The $\Delta G^{\text {exc }}$ values display a minimum $\left(-1100 \mathrm{~J} \mathrm{~mol}^{-1}\right)$ at $50 \mathrm{mN} \mathrm{m}^{-1}$ in $X_{\mathrm{PSM}}=0.3$. Overall, the minimum $\Delta G^{\text {exc }}$ value appears at $X_{\mathrm{PSM}}=0.5$, from 5 to $35 \mathrm{mN} \mathrm{m}^{-1}$, and it appears to shift to $X_{\mathrm{PSM}}=0.3$ above $35 \mathrm{mN} \mathrm{m}^{-1}$. Compared with the PSM/SITO system, the $\Delta G^{\text {exc }}$ values for the PSM/ SG system form a small negative convex curve. It was suggested that the D-glucose moiety of SG prevents interaction between the two molecules. $\Delta G^{\text {exc }}-X_{\text {PSM }}$ curves for the PSM/ SGP system are shown in Fig. 5(C). The maximum $\Delta G^{\text {exc }}$ values, over the whole surface pressure range, appear at $X_{\mathrm{PSM}}=0.7$, which is the same as for the PSM/SITO system (Fig. 5(A)). In addition, $\Delta G^{\text {exc }}$ values are fully negative and attain a minimum at $X_{\mathrm{PSM}}=0.7\left(-1700 \mathrm{~J} \mathrm{~mol}^{-1}\right)$. When this result is compared with the result for the PSM/SG system, the $\Delta G^{\text {exc }}$ values for the PSM/SGP system are a negative convex shape. The palmitoyl group incorporated into SG may account for this result as it enhances the cohesive attraction of double-saturated acyl chains in PSM.

Collectively, the $\Delta G^{\text {exc }}$ values (Fig. 5) and additivities in the area per molecule (Fig. 4) revealed that PSM interacts most with SITO, followed by SGP and SG, that is, the interaction with PSM is reduced by incorporation of D-glucose into SITO, whereas it is recovered by introduction of a palmitoyl group into SG.

\subsection{Compression modulus values}

To investigate the influence of the respective phytosterol derivatives on PSM monolayers, a compressibility modulus (or dilatational elasticity modulus, $\mathrm{Cs}^{-1}$ ) was calculated using Eq. (10):

$$
\mathrm{Cs}^{-1}=-A\left(\frac{\partial \pi}{\partial A}\right)_{\mathrm{T}}
$$

The $\mathrm{Cs}^{-1}$ value gives information on the packed state of monolayers upon compression. A high compressibility modulus (or low compressibility) indicates tight packing and a large cohesive force between the components. Figure 6 shows the maximum compressibility modulus $\left(\mathrm{Cs}^{-1}\right)$ as a 

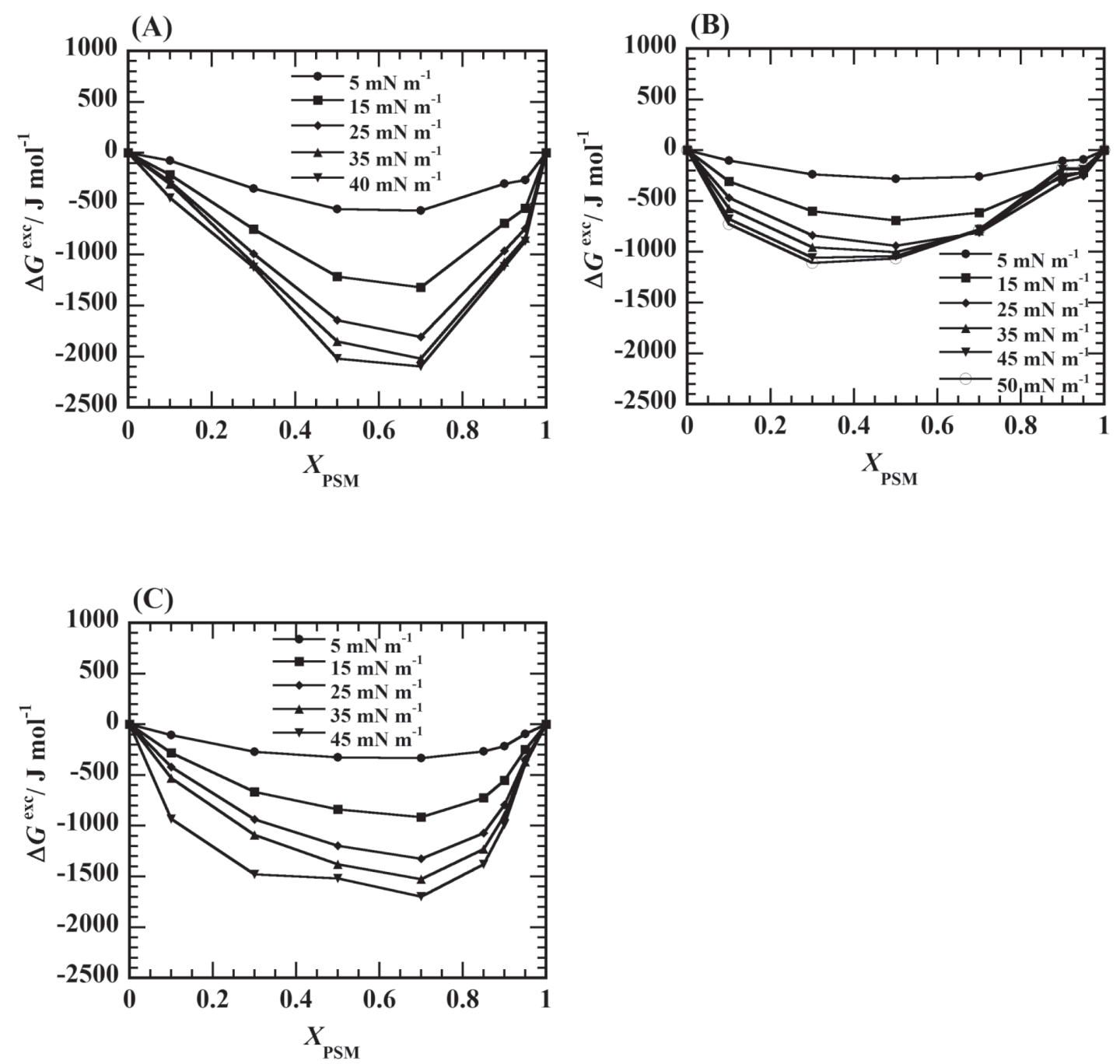

Fig. 5 Excess Gibbs free energy of mixing for (A) PSM/SITO, (B) PSM/SG, and (C) PSM/SGP monolayers as a function of $X_{\mathrm{PSM}}$ at different surface pressures. $\Delta G^{\text {exc }}$ value was calculated from Eq. (9).

function of $X_{\mathrm{PSM}}$ for the binary monolayers. At $0 \leq X_{\mathrm{PSM}} \leq$ 0.1 , the $\mathrm{Cs}^{-1}$ values of the SG system decrease dramatically, whereas those of the SITO and SGP systems increase. The latter result indicates that a small amount of PSM promotes monolayer packing in the PSM/SITO and PSM/SGP systems. Further addition of PSM to phytosterol derivative monolayers induces a decrease in $\mathrm{Cs}^{-1}$ values at $0.1 \leq X_{\mathrm{PSM}}$ $\leq 0.9$, and the $\mathrm{Cs}^{-1}$ values attain a minimum at $X_{\mathrm{PSM}}=0.9$ in all three systems. This result suggests that a small amount of phytosterol derivative perturbs the ordering of the acyl chains in PSM, which reflects a disordering effect of sterols $^{29)}$. Similar behavior was observed when SM and DPPC were mixed with a small molar fraction $(\sim 0.1)$ of androsterol, 6-ketocholesterol ${ }^{52)}$, CHOL, SITO, and stigmasterol $^{29)}$. Considering the deviations of $\mathrm{Cs}^{-1}-X_{\mathrm{PSM}}$ from linearity between pure components (Fig. 6), the $\mathrm{Cs}^{-1}$ values of SITO and SG display positive and negative deviations, respectively. These are especially remarkable at $X_{\mathrm{PSM}} \leq 0.5$,

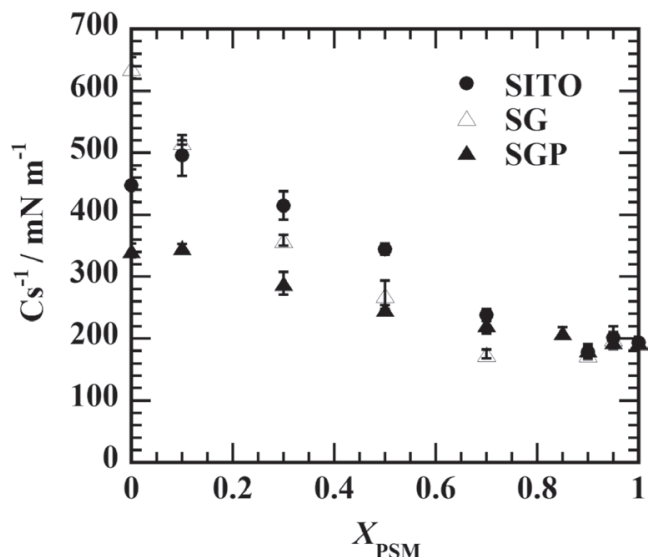

Fig. 6 The maximum compression modulus $\left(\mathrm{Cs}^{-1}\right)$ values of the binary PSM/SITO, PSM/SG, and PSM/SGP monolayers as a function of $X_{\mathrm{PSM}}$. $\mathrm{Cs}^{-1}$ values were calculated from Eq. (10). 
even though those of SGP are nearly at the tie line. That is, addition of PSM into SITO and SG monolayers promotes more and less ordered states of binary monolayers, respectively. It is postulated that SITO can swing its tail moiety (steroid backbone) more than SG at the air-water interface, because the head group of SITO (hydroxyl group) is smaller than that of SG (D-glucose moiety). Therefore, for the PSM/ SITO system, the motion of SITO is thought to be limited by PSM addition, promoting a more ordered state. In contrast, for the PSM/SG system, the limited motion of SG induced by incorporated D-glucose is likely promoted by the addition of PSM, which causes a less ordered state of monolayers.

\subsection{Two-dimensional phase diagram}

Two-dimensional phase diagrams for the binary systems at $298.2 \mathrm{~K}$ were constructed by plotting values of $\pi^{\mathrm{eq}}$ and $\pi^{\mathrm{c}}$ as a function of $X_{\mathrm{PSM}}$ (Fig. 7). The results show that the $\pi^{\mathrm{eq}}$ value negatively deviates at $0.95 \leq X_{\mathrm{PSM}} \leq 1$ for the PSM/ SITO (Fig. $7(\mathrm{~A})$ ) system and at $0.9 \leq X_{\mathrm{PSM}} \leq 1$ for the PSM/
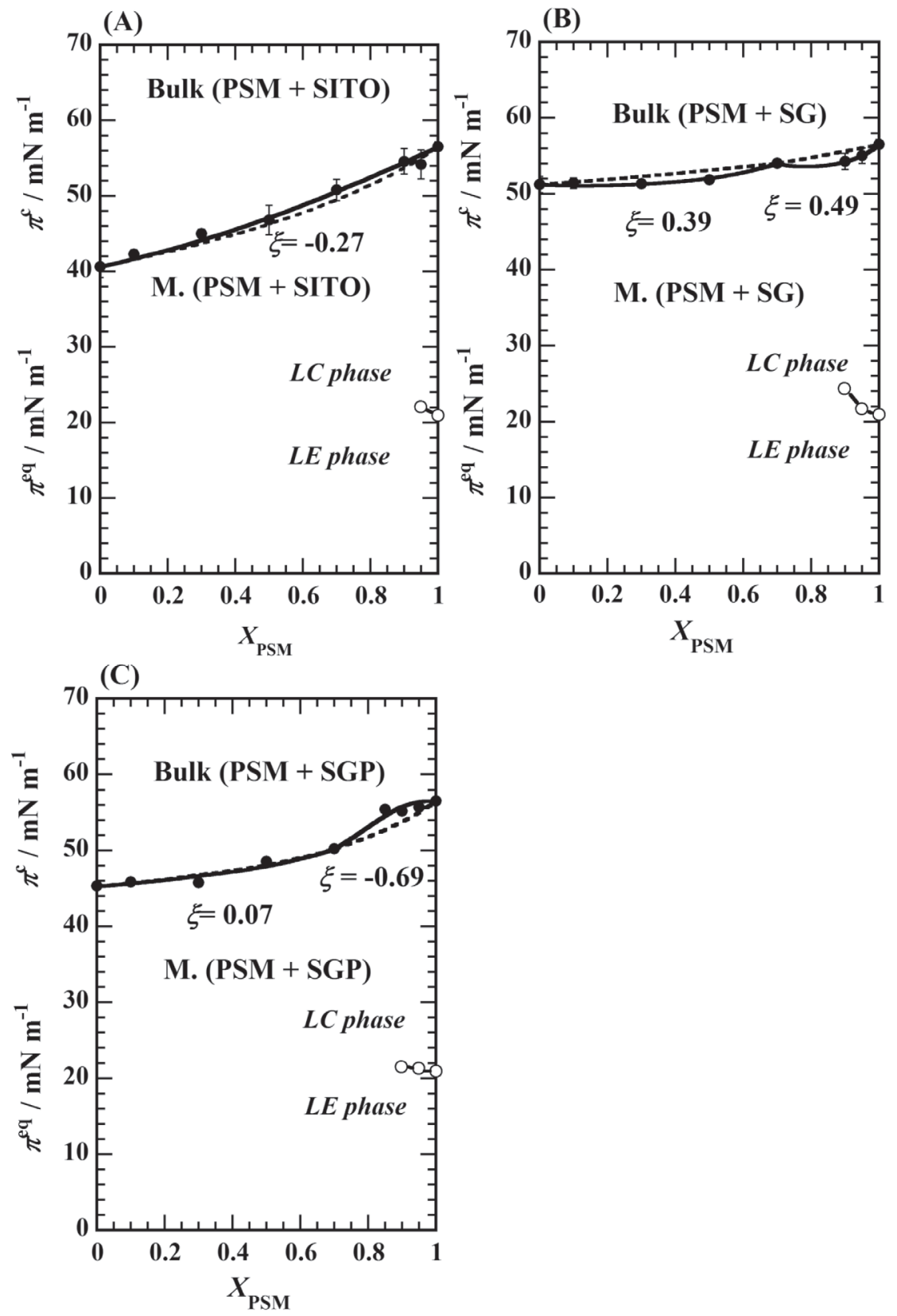

Fig. 7 Two-dimensional phase diagrams based on the variation of the transition pressure ( $\pi^{\mathrm{eq}}$ : open circle) and collapse pressure ( $\pi^{\mathrm{c}}$ : solid circle) as a function of $X_{\mathrm{PSM}}$; (A) PSM/SITO, (B) PSM/SG, and (C) PSM/SGP. The dashed lines were calculated according to the Eq. (11). M. indicates a monolayer state. Bulk indicates a solid phase. 
SG (Fig. 7(B)) and PSM/SGP (Fig. 7(C)) systems. Regarding monolayer collapse, the experimental values of $\pi^{\mathrm{c}}$ vary with $X_{\mathrm{PSM}}$ for all systems. Hence, the two components in the three binary systems are miscible in the monolayer state.

Assuming that the above binary monolayers behave as regular surface mixtures with a hexagonal lattice, the coexistence phase boundary between the ordered monolayer (two-dimensional phase) and bulk phase (three-dimensional phase) of the molecules spread on the surface can be theoretically simulated by the Joos equation ${ }^{53)}$ :

$$
\begin{aligned}
1= & x_{1}^{\mathrm{s}} \exp \left\{\left(\pi_{\mathrm{m}}^{\mathrm{c}}-\pi_{1}^{\mathrm{c}}\right) \omega_{1} / k T\right\} \exp \left\{\xi\left(x_{2}^{\mathrm{s}}\right)^{2}\right\} \\
& +x_{2}^{\mathrm{s}} \exp \left\{\left(\pi_{\mathrm{m}}^{\mathrm{c}}-\pi_{2}^{\mathrm{c}}\right) \omega_{2} / k T\right\} \exp \left\{\xi\left(x_{1}^{\mathrm{s}}\right)^{2}\right\}
\end{aligned}
$$

where $x_{1}^{\mathrm{s}}$ and $x_{2}^{\mathrm{s}}$ denote the respective molar fractions in the two-component monolayers of components 1 and 2 , respectively, and $\pi_{1}^{\mathrm{c}}$ and $\pi_{2}^{\mathrm{c}}$ are the respective collapse pressures of pure component 1 and $2, \pi_{\mathrm{m}}^{\mathrm{c}}$ is the collapse pressure of the two-component monolayer at a given composition of $x_{1}^{\mathrm{s}}\left(\right.$ or $\left.x_{2}^{\mathrm{s}}\right), \omega_{1}$ and $\omega_{2}$ are the corresponding areas per molecule at the collapse points, $\xi$ is the interaction parameter, and $k T$ is the product of the Boltzmann constant and temperature $(\mathrm{K})$. The solid curve at higher surface pressures was obtained by adjusting the interaction parameter in Eq. (11) for the best fit of the experimental $\pi^{c}$ values. As shown in Fig. 7(A), the PSM/SITO system shows $\xi=-0.27$. Negative interaction parameters imply domination of the interaction between different molecules relative to interactions between like molecules. Accordingly, this suggests that the intermolecular interaction of PSM-SITO is stronger. The $\xi$ value for the PSM/SG and PSM/SGP systems is divided into two regions at $X_{\mathrm{PSM}}=0.7$. Contrary to the PSM/SITO system, the $\xi$ value for the PSM/SG system is positive $\left(\xi=0.39\right.$ at $0 \leq X_{\mathrm{PSM}} \leq 0.7$ and $\xi=0.49$ at $\left.0.7 \leq X_{\mathrm{PSM}} \leq 1\right)$ over all molar fractions (Fig. $7(\mathrm{~B})$ ), that is, the intermolecular interaction of PSM-PSM or SG-SG is estimated to be stronger than that of PSM-SG. As for the $\mathrm{PSM} / \mathrm{SGP}$ system, the $\xi$ value is both positive $(\xi=0.07$ at 0 $\left.\leq X_{\mathrm{PSM}} \leq 0.7\right)$ and negative $\left(\xi=-0.69\right.$ at $\left.0.7 \leq X_{\mathrm{PSM}} \leq 1\right)$, as shown in Fig. $7(\mathrm{C})$. The miscibility manner at $0.7 \leq X_{\mathrm{PSM}} \leq$ 1 in the PSM/SGP system is the opposite of that seen in the PSM/SG system. Thus, this result reveals that the incorporation of a palmitoyl group into SG plays an important role in regulating its miscibility manner with PSM at $0.7 \leq X_{\mathrm{PSM}} \leq$ 1.

The interaction energy $(\Delta \varepsilon)$ is given as follows:

$$
-\Delta \varepsilon=\xi R T / z
$$

where $z$ is the number of nearest neighbors (equal to 6 ) in a hexagonal packing; the interaction energy is also described as $-\Delta \varepsilon=\varepsilon_{12}-\left(\varepsilon_{11}+\varepsilon_{22}\right) / 2^{53)}$; and $\varepsilon_{i j}$ represents the potential energy of interactions between components $i$ and $j$. Table 2 shows the interaction parameters $(\xi)$ and corresponding interaction energies $(\Delta \varepsilon)$ calculated using Eq. (11) and (12), respectively. The interaction energies were calculated to be $116 \mathrm{~J} \mathrm{~mol}^{-1}(\xi=-0.27)$ for the PSM/SITO system and $-161 \mathrm{~J} \mathrm{~mol}^{-1}(\xi=0.39)$ and $-202 \mathrm{~J} \mathrm{~mol}^{-1}(\xi=$ $0.49)$ for the PSM/SG system. The PSM/SGP system had interaction energies of $-29 \mathrm{~J} \mathrm{~mol}^{-1}(\xi=0.07)$ and $285 \mathrm{~J} \mathrm{~mol}^{-1}$ $(\xi=-0.69)$. It was found that the two components were miscible, because the interaction energies were less than $2 R T\left(4958.7 \mathrm{~J} \mathrm{~mol}^{-1}\right)$. The interaction energy for the PSM/ SITO system is approximately half that of the PSM/CHOL $\operatorname{system}\left(\xi=-0.59,244 \mathrm{~J} \mathrm{~mol}^{-1}\right)^{34)}$ (Table 2). This can be accounted for by the structural difference between CHOL and SITO, which was described previously in the section on excess Gibbs free energy. Furthermore, the $\Delta \varepsilon$ value between representative phospholipids DPPC and CHOL has been reported to be zero ${ }^{53}$. Their interaction behavior corresponds to our results, showing that the $\Delta \varepsilon$ values between PSM and phytosterol derivatives (CHOL, SITO, SG, and SGP) are too small (Table 2). Thus, the interaction between phospholipids or sphingolipids and phytosterol derivatives is regulated by the interactions between saturated aliphatic chains and steroid backbones.

\subsection{Morphological analysis}

In situ morphological observations were carried out using BAM and FM. Figure 8 shows FM images of single

Table 2 Interaction parameter $(\xi)$ and interaction energy $(\Delta \varepsilon)$ for the binary systems calculated by the Eqs. (11) and (12), respectively.

\begin{tabular}{lcrc}
\hline \multicolumn{1}{c}{ System } & $X_{\mathrm{PSM}}$ & \multicolumn{1}{c}{$\xi$} & $\Delta \varepsilon\left(\mathrm{J} \mathrm{mol}^{-1}\right)$ \\
\hline $\mathrm{PSM} / \mathrm{SITO}$ & $0 \leq X_{\mathrm{PSM}} \leq 1$ & $-0.27 \pm 0.20$ & $116 \pm 70$ \\
\hline \multirow{2}{*}{$\mathrm{PSM} / \mathrm{SG}$} & $0 \leq X_{\mathrm{PSM}} \leq 0.7$ & $0.39 \pm 0.07$ & $-161 \pm 30$ \\
& $0.7 \leq X_{\mathrm{PSM}} \leq 1$ & $0.49 \pm 0.20$ & $-202 \pm 70$ \\
\hline \multirow{2}{*}{$\mathrm{PSM} / \mathrm{SGP}$} & $0 \leq X_{\mathrm{PSM}} \leq 0.7$ & $0.07 \pm 0.02$ & $-29 \pm 10$ \\
& $0.7 \leq X_{\mathrm{PSM}} \leq 1$ & $-0.69 \pm 0.06$ & $285 \pm 25$ \\
\hline $\mathrm{PSM} / \mathrm{CHOL}^{34)}$ & $0 \leq X_{\mathrm{PSM}} \leq 1$ & $-0.59 \pm 0.06$ & $244 \pm 25$ \\
\hline
\end{tabular}


(A)

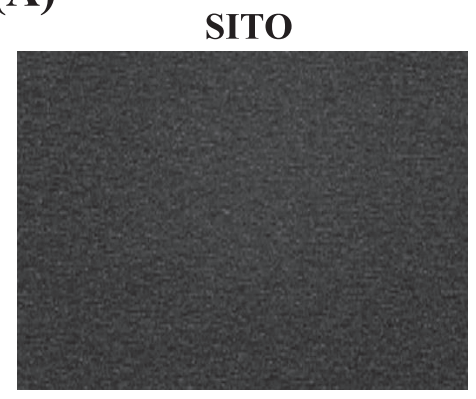

\section{PSM}

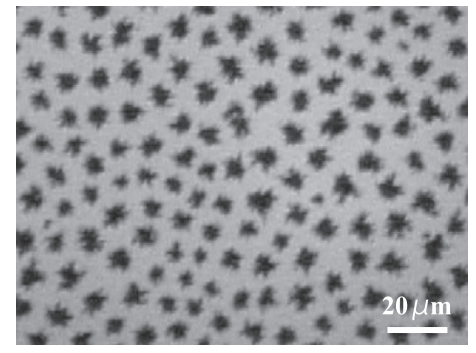

(B)

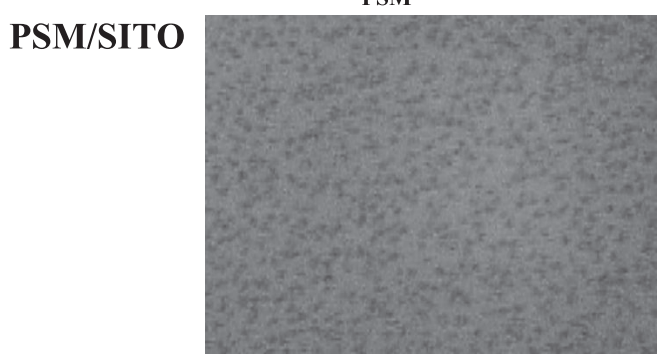

PSM/SG

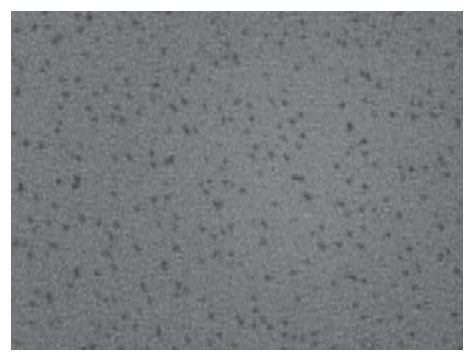

PSM/SGP

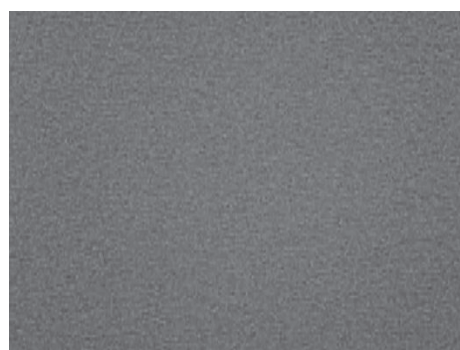

SG
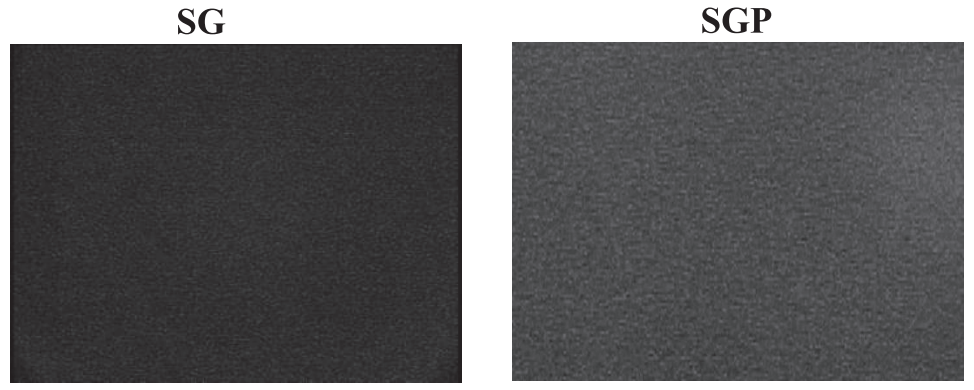
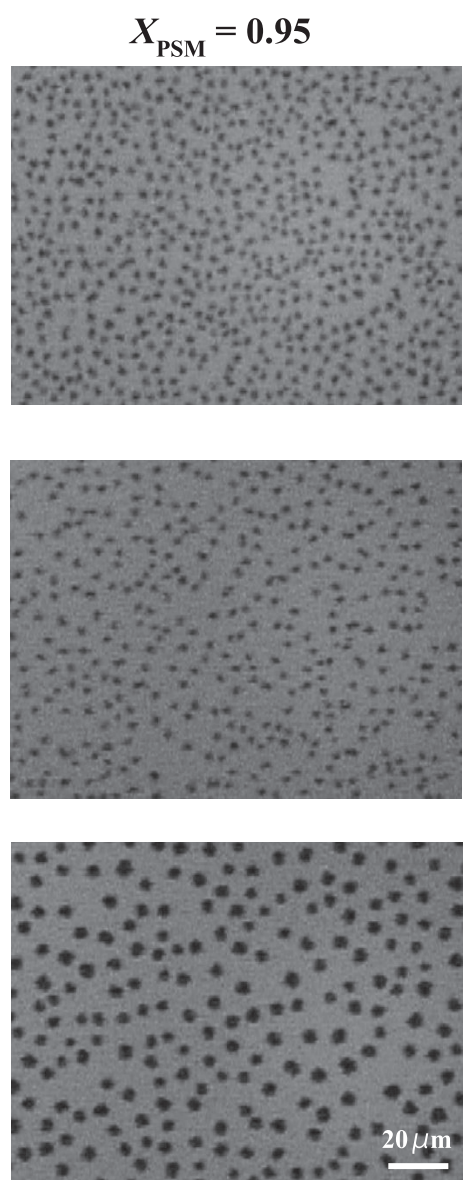

Fig. 8 Fluorescence micrographs of (A) pure SITO, SG, SGP and PSM and (B) binary PSM/SITO, PSM/SG, and PSM/SGP monolayers $\left(X_{\mathrm{PSM}}=0.9\right.$ and 0.95$)$ at $25 \mathrm{mN} \mathrm{m}^{-1}$ on $0.02 \mathrm{M}$ Tris buffer with $0.13 \mathrm{M} \mathrm{NaCl}(\mathrm{pH} 7.4)$ at $298.2 \mathrm{~K}$. The monolayers contain $1 \mathrm{~mol} \%$ of the fluorescent probe (NBD-PC). The scale bars of the Fig. 8 (B) in the lower-right represent $20 \mu \mathrm{m}$. 


\section{S. Sakamoto, H. Nakahara and O. Shibata}

(SITO, SG, and SGP) and binary (PSM/SITO, PSM/SG, and $\mathrm{PSM} / \mathrm{SGP}$ ) monolayers at $X_{\mathrm{PSM}}=0.9$ and 0.95 on $0.02 \mathrm{M}$ Tris buffer with $0.13 \mathrm{M} \mathrm{NaCl}(\mathrm{pH} 7.4)$ at $25 \mathrm{mN} \mathrm{m}^{-1}$. A fluorescent probe in the FM measurement (here, NBD-PC) can selectively dissolve in LE phases. Thus, bright and dark domains in the FM images correspond to the LE and LC phases, respectively.

For the FM images of pure SITO, SG, and SGP(Fig. 8 (A)), homogeneous LC phases (dark contrast) are observed over the whole range of surface pressures. Interestingly, the FM images of the pure SG monolayer are darker than the images of the pure SITO and SGP monolayers. This means that the SG monolayer is more condensed than the pure SITO and SGP monolayers. The difference in contrast was supported by the corresponding BAM. This is also in agreement with the results in Fig. 6.

Similar to the FM images for the pure individuals, the FM images of the binary PSM/SITO, PSM/SG, and PSM/SGP systems (Fig. 8(B)) featured uniform LC phases (dark contrast) at $0.1 \leq X_{\mathrm{PSM}} \leq 0.7$ for the $\mathrm{PSM} / \mathrm{SITO}$ and $\mathrm{PSM} / \mathrm{SG}$ systems and $0.1 \leq X_{\mathrm{PSM}} \leq 0.9$ for the PSM/SGP system (data not shown). At $X_{\mathrm{PSM}} \geq 0.9$ for the PSM/SITO and PSM/SG systems, the microdomains of the LC phases start to form at $\sim 24$ and $25 \mathrm{mN} \mathrm{m}^{-1}$, respectively, although the $\pi^{\mathrm{eq}}$ value for the PSM/SITO system from the $\pi-A$ isotherms is not confirmed (Fig. 3(A)). In the FM images at $X_{\mathrm{PSM}} \geq 0.95$ for the PSM/SGP system, the microdomains emerge at $\sim 22$ $\mathrm{mN} \mathrm{m}^{-1}$. These morphological results reveal that PSM and phytosterol derivatives (SITO, SG, and SGP) are miscible, since the microdomains emerge at a different $\pi^{\mathrm{eq}}$ value from $\operatorname{PSM}\left(\sim 21 \mathrm{mN} \mathrm{m}^{-1}\right)$. Also, the addition of a small amount of phytosterol derivative induces variations in the shape and size of the microdomains compared with PSM (Fig. 8), which is further evidence that PSM interacts with phytosterol derivatives. However, the correlation between domain size and thermodynamic parameters did not agree with the FM analysis. Nanometric methodologies, such as atomic force microscopy, may be helpful in investigating the correlation in more detail ${ }^{54,55)}$.

\section{Conclusions}

The intermolecular interaction between PSM and phytosterol derivatives of SITO, SG, and SGP has been systematically investigated employing the Langmuir monolayer. A thermodynamic analysis based on $\pi-A$ and $\Delta V-A$ isotherms revealed that favorable interactions of the derivatives with PSM occurred in the following order: SITO $>$ SGP $>$ SG. This suggested that the interaction with PSM is interrupted by the incorporation of the D-glucose moiety into SITO and is recovered by the introduction of a palmitoyl group into the D-glucose of SG. However, the degree of PSM-SGP interaction is less than that seen for PSM-SITO. The polar head groups of PSM and the phytosterol derivatives, along with the hydrophobic tail groups, strongly influence surface potential. The Demchak and Fort model was applied to analyze the surface potential data, and from those data the dipole moments of the polar head groups were determined to be $2.5 \mathrm{D}$ for SG, $4.2 \mathrm{D}$ for SGP, and 1.3 D for PSM. Furthermore, the compressibility modulus $\left(\mathrm{Cs}^{-1}\right)$ values showed that the size of the head groups influences the packing manner in the binary monolayer. Subsequently, miscibility between the two components was evaluated by constructing a two-dimensional phase diagram based on $\pi^{c}$ and $\pi^{\text {eq }}$ values. As a result, the two components are found to be miscible with each other, but the miscibility is different in terms of interaction parameters and energies. Lipid rafts are mainly composed of SM and $\mathrm{CHOL}^{7,56,57)}$ Considering that phytosterols lower the level of CHOL in plasma by competitive inhibition of CHOL absorption ${ }^{14,16,22,23)}$, and that the PSM-SITO interaction is almost the same as the PSM-CHOL interaction, these findings reveal that SITO does not have a negative effect on biological membranes. This is true even if it is competitively absorbed into the intestine instead of CHOL. The present study on the penetration effects of the D-glucose moiety and palmitoyl group on the PSM monolayer may provide useful information for estimating three-dimensional interactions, such as those of a variety of sterol derivatives with the PSM biomembrane, which are of great interest in health care.

\section{Acknowledgements}

The research in this paper was supported by the Japan Food Chemical Research Foundation. This work was also funded by a Grant-in-Aid for Young Scientists (B) [25750167] of the Japan Society for the Promotion of Science(JSPS).

\section{References}

1) Rajendran, L.; Simons, K. Lipid rafts and membrane dynamics. J. Cell Sci. 118, 1099-1102 (2005).

2) Simons, K.; Ikonen, E. Functional rafts in cell membranes. Nature 387, 569-572 (1997).

3) Simons, K.; Toomre, D. Lipid rafts and signal transduction. Nat. Rev. Mol. Cell. Biol. 1, 31-39 (2000).

4) Brown, D. A. Seeing is believing: visualization of rafts in model membranes. Proc. Natl. Acad. Sci. 98, 10517-10518(2001).

5) Ipsen, J. H.; Karlstrom, G.; Mouritsen, O. G.; Wennerstrom, H.; Zuckermann, M. J. Phase equilibria in the phosphatidylcholine-cholesterol system. Biochim. Biophys. Acta 905, 162-172 (1987).

6) Recktenwald, D. J.; McConnell, H. M. Phase equilibria 
in binary mixtures of phosphatidylcholine and cholesterol. Biochemistry 20, 4505-4510 (1981).

7) Sankaram, M. B.; Thompson, T. E. Interaction of cholesterol with various glycerophospholipids and sphingomyelin. Biochemistry 29, 10670-10675(1990).

8) Munro, S. Lipid rafts: elusive or illusive? Cell 115, 377388 (2003).

9) Parpal, S.; Karlsson, M.; Thorn, H.; Stralfors, P. Cholesterol depletion disrupts caveolae and insulin receptor signaling for metabolic control via insulin receptor substrate-1, but not for mitogen-activated protein kinase control. J. Biol. Chem. 276, 9670-9678(2001).

10) Zajchowski, L. D.; Robbins, S. M. Lipid rafts and little caves. Compartmentalized signalling in membrane microdomains. Eur. J. Biochem. 269, 737-752 (2002).

11) Holthuis, J. C.; van Meer, G.; Huitema, K. Lipid microdomains, lipid translocation and the organization of intracellular membrane transport(Review). Mol. Membr. Biol. 20, 231-241 (2003).

12) Smart, E. J.; Ying, Y.; Donzell, W. C.; Anderson, R. G. A role for caveolin in transport of cholesterol from endoplasmic reticulum to plasma membrane. J. Biol. Chem. 271, 29427-29435 (1996).

13) Fedida-Metula, S.; Elhyany, S.; Tsory, S.; Segal, S.; Hershfinkel, M.; Sekler, I.; Fishman, D. Targeting lipid rafts inhibits protein kinase $\mathrm{B}$ by disrupting calcium homeostasis and attenuates malignant properties of melanoma cells. Carcinogenesis 29, 1546-1554 (2008).

14) Pollak, O. J. Reduction of blood cholesterol in man. Circulation 7, 702-706 (1953).

15) Ostlund, R. E. J. Phytosterols in human nutrition. Ann. Rev. Nut. 22, 533-549(2002).

16) Tilvis, R. S.; Miettinen, T. A. Serum plant sterols and their relation to cholesterol absorption. Am. J. Clin. Nutr. 43, 92-97 (1986).

17) Furt, F.; Lefebvre, B.; Cullimore, J.; Bessoule, J. J.; Mongrand, S. Plant Lipid Rafts. Plant Signal. Behav. 2, 508-511(2007).

18） Grille, S.; Zaslawski, A.; Thiele, S.; Plat, J.; Warnecke, D. The functions of steryl glycosides come to those who wait: Recent advances in plants, fungi, bacteria and animals. Prog. Lipid Res. 49, 262-288(2010).

19) Kovganko, N. V.; Kashkan Z. N. Sterol glycosides and acylglycosides. Chem. Nat. Compd. 35, 479-497 (1999).

20) Lepage, M. Isolation and characterization of an esterified form of steryl glucoside. J. Lipid Res. 5, 587-592 (1964).

21) Cacas, J. L.; Furt, F; Le Guédard, M.; Schmitter, J. M.; Buré, C.; Gerbeau-Pissot, P.; Moreau, P.; Bessoule, J. J.; Simon-Plas, F.; Mongrand, S. Lipids of plant membrane rafts. Prog. Lipid Res. 51, 272-299 (2012).

22) Lin, X.; Ma, L.; Racette, S. B.; Anderson, Spearie, C. L.;
Ostlund, R. E. Jr. Phytosterol glycosides reduce cholesterol absorption in humans. Am. J. Physiol. Gastrointest. Liver Physiol. 296, G931-935 (2009).

23) Lin, X.; Ma, L.; Moreau, R. A.; Ostlund, R. E. Jr. Glycosidic bond cleavage is not required for phytosteryl glycoside-induced reduction of cholesterol absorption in mice. Lipids 46, 701-708(2011).

24) Bruckdorfer, K. R.; Demel, R. A.; De Gier, J.; van Deenen, L. L. The effect of partial replacements of membrane cholesterol by other steroids on the osmotic fragility and glycerol permeability of erythrocytes. Biochim. Biophys. Acta 183, 334-345 (1969).

25) Naito, Y.; Konishi, C.; Ohara, N. Blood coagulation and osmolar tolerance of erythrocytes in stroke-prone spontaneously hypertensive rats given rapeseed oil or soybean oil as the only dietary fat. Toxicol. Lett. 116, 209-215 (2000).

26) Sabatini, K.; Mattila, J. P.; Kinnunen, P. K. Interfacial behavior of cholesterol, ergosterol, and lanosterol in mixtures with DPPC and DMPC. Biophys. J. 95, 23402355 (2008).

27) Hąc-Wydro, K. The replacement of cholesterol by phytosterols and the increase of total sterol content in model erythrocyte membranes. Chem. Phys. Lipids 163, 689-697 (2010).

28) Hąc-Wydro, K. Langmuir monolayers studies on the relationship between the content of cholesterol in model erythrocyte membranes and the influence of $\beta$-sitosterol. Colloids and Surfaces B: Biointerfaces 91, 226-233 (2012).

29) Hąc-Wydro, K.; Dynarowicz-Łątka, P. The Impact of Sterol Structure on the Interactions with Sphingomyelin in Mixed Langmuir Monolayers. J. Phys. Chem. B 112, 11324-11332 (2008)

30) H ąc-Wydro, K.; Wydro, P.; Dynarowicz-Łątka, P.; Paluch, M. Cholesterol and phytosterols effect on sphingomyelin/phosphatidylcholine model membranes-Thermodynamic analysis of the interactions in ternary monolayers. J. Colloid Interface Sci. 329, 265-272 (2009).

31) Nakahara, H.; Lee, S.; Krafft, M. P.; Shibata, O. Fluorocarbon-hybrid pulmonary surfactants for replacement therapy - A Langmuir monolayer study. Langmuir 26, 18256-18265 (2010).

32) Nakahara, H.; Lee, S.; Shoyama, Y.; Shibata, O. The role of palmitic acid in pulmonary surfactant systems by Langmuir monolayer study: Lipid-peptide interactions. Soft Matter 7, 11351-11359(2011).

33) Nakahara, H.; Shibata, O.; Moroi, Y. Examination of surface adsorption of cetyltrimethylammonium bromide and sodium dodecyl sulfate. J. Phys. Chem. B 115, 9077-9086 (2011).

34) Sakamoto, S.; Nakahara, H.; Uto, T.; Shoyama, Y.; Shibata, O. Investigation of interfacial behavior of glycyr- 


\section{S. Sakamoto, H. Nakahara and O. Shibata}

rhizin with a lipid raft model via a Langmuir monolayer study. Biochim. Biophys. Acta 1828, 1271-1283 (2013).

35) Walker, B. W.; Manhanke, N.; Stine, K. J. Comparison of the interaction of tomatine with mixed monolayers containing phospholipid, egg sphingomyelin, and sterols. Biochim. Biophys. Acta 1778, 2244-2257 (2008).

36) Maget-Dana, R. The monolayer technique: a potent tool for studying the interfacial properties of antimicrobial and membrane-lytic peptides and their interactions with lipid membranes. Biochim. Biophys Acta 1462, 109-140 (1999).

37) Vogel, V.; Möbius, D. Local surface potentials and electric dipole moments of lipid monolayers: contributions of the water/lipid and the lipid/air interfaces. J. Colloid Interface Sci. 126, 408-420 (1988).

38) Hoda, K.; Ikeda, Y.; Kawasaki, H.; Yamada, K.; Higuchi, R.; Shibata, O. Mode of interaction of ganglioside Langmuir monolayer originated from echinoderms: Three binary systems of ganglioside/DPPC, ganglioside/DMPE, and ganglioside/cholesterol. Colloids and Surfaces B: Biointerfaces 52, 57-75 (2006).

39) Shah, D. O.; Schulman, J. H. Influence of calcium, cholesterol, and unsaturation on lecithin monolayers. $J$. Lipid Res. 8, 215-226 (1967).

40) Marsden, J.; Schulman, J. H. Molecular interaction in monolayers. Trans. Faraday Soc. 34, 748-758(1938).

41) Demchak, R. J.; Fort Jr, T. Surface dipole moments of close-packed un-ionized monolayers at the air-water interface. J. Colloid Interface Sci. 46, 191-202 (1974).

42) Davies, J. T.; Rideal, E. K., Interfacial Phenomena. 2nd ed.; Academic Press: New York: pp 71 (1963).

43) Vogel, V.; Möbius, D. Hydrated polar groups in lipid monolayers: Effective local dipole moments and dielectric properties. Thin Solid Films 159, 73-81 (1988).

44) Taylor, D. M.; De Oliveira Jr, O. N.; Morgan, H. Models for interpreting surface potential measurements and their application to phospholipid monolayers. J. Colloid Interface Sci. 139, 508-518(1990).

45) Petrov, J. G; Polymeropoulos, E. E.; Möhwald, H. Three-capacitor model for surface potential of insoluble monolayers. J. Phys. Chem. A 100, 9860-9869 (1996).

46) a) Nyrén, V.; Back, E. The ionization constant, solubility product and solubility of abietic and dehydroabietic acid. Acta Chem. Scand. 12, 1305-1311 (1958).

b) Caspers, J.; Goormaghtigh, E.; Ferreira, J.; Brasseur, R.; Vandenbranden, M.; Ruysschaert, J. M. Acido-basic properties of lipophilic substances: A surface potential approach. J. Colloid Interface Sci. 91, 546-551
(1983).

c) Taylor, D. M.; Oliveira, O. N.; Morgan, H. Surface potential of monolayers formed on weak acidic electrolytes. Chem. Phys. Lett. 161, 147-150 (1989).

d) Calvez, E. L; Blaudez, D.; Buffeteau, T.; Desbat, B. Effect of cations on the dissociation of arachidic acid monolayers on water studied by polarization-modulated infrared reflection-absorption spectroscopy. Langmuir 17, 670-674(2001).

47) Bernett, M. K.; Jarvis, N. L.; Zisman, W. A. Properties of monolayers of $\omega$-monohalogenated fatty acids and alcohols adsorbed on water ${ }^{1}$. J. Phys. Chem. 68, 35203529 (1964).

48) Smyth, C. P. Dielectric Behavior and Structure, McGraw-Hill, New York, 1955.

49) Bernett, M. K.; Zisman, W. A. The behavior of monolayers of progressively fluorinated fatty acids adsorbed on water. J. Phys. Chem. 67, 1534-1540 (1963).

50) Gaines, G. L., Insoluble Monolayers at Liquid/Gas Interfaces, Interscience, New York, (1966).

51) Goodrich, F. C. Proceedings of the 2nd International Congress on Surface Activity, Butterworth \& Co., London, (1957).

52) Li, X. M.; Momsen, M. M.; Brockman, H. L.; Brown, R. E. Sterol structure and sphingomyelin acyl chain length modulate lateral packing elasticity and detergent solubility in model membranes. Biophys. J. 85, 3788-3801 (2003).

53) Joos, P.; Demel, R. A. The interaction energies of cholesterol and lecithin in spread mixed monolayers at the air-water interface. Biochim. Biophys. Acta 183, 447-457 (1969).

54) Yokoyama, H.; Nakahara, H.; Nakagawa, T.; Shimono, S.; Sueishi, K.; Shibata, O. Miscibility behavior of twocomponent monolayers at the air-water interface: Perfluorocarboxylic acids and DMPE. J. Colloid Interface Sci. 337, 191-200 (2009).

55) Nakahara, H.; Krafft, M. P.; Shibata, A.; Shibata, O. Interaction of a partially fluorinated alcohol $(F 8 H 11 \mathrm{OH})$ with biomembrane constituents in two-component monolayers. Soft Matter 7, 7325-7333 (2011).

56) Barenholz, Y.; Thompson T. E. Sphingomyelins in bilayers and biological membranes. Biochim. Biophys. Acta 604, 129-158(1980).

57) Björkbom, A.; Róg, T.; Kankaanpää, P.; Lindroos, D.; Kaszuba, K.; Kurita, M.; Yamaguchi, S.; Yamamoto, T.; Jaikishan, S.; Paavolainen, L.; Päivärinne, J.; Nyholm, T. K. M.; Katsumura, S.; Vattulainen, I.; Slotte, J. P. Nand O-methylation of sphingomyelin markedly affects its membrane properties and interactions with cholesterol. Biochim. Biophys. Acta 1808, 1179-1186 (2011). 